\title{
Synthesis of Novel Fluorine Substituted Isolated and Fused Heterobicyclic Nitrogen Systems Bearing 6-(2'-Phosphorylanilido)- 1,2,4-Triazin-5-One Moiety as Potential Inhibitor towards HIV-1 Activity
}

\author{
Reda M. Abdel-Rahman, Mohammed S. T. Makki, Abeer N. Al-Romaizan* \\ Department of Chemistry, Faculty of Science, King Abdul Aziz University, Jeddah, KSA \\ Email: ar-orkied@hotmail.com
}

Received 13 September 2014; revised 29 October 2014; accepted 14 November 2014

Copyright (C) 2014 by authors and Scientific Research Publishing Inc.

This work is licensed under the Creative Commons Attribution International License (CC BY).

http://creativecommons.org/licenses/by/4.0/

(c) ()) Open Access

\begin{abstract}
Novel 6-(5'-fluoro-2'-diphenylphosphorylanilido)-3-hydrazino-1,2,4-trizin-5 (2H) one (3) is achieved from hydrozinolysis of the corresponding 3-thioxo-analoges 2 . Compound 2 is also obtained from phosphorylation of 6-(5'-fluoro-2'-aminophenyl)-3-thioxo-1,2,4-triazin-5(2H) one (1). Novel fluorine substituted isolated and/or fused heterobicyclic nitrogen systems bearing and/or containing, 6-phosphoryl anilido-1,2,4-trizin-5 $(2 \mathrm{H})$ one moiety $(4-22)$ have been synthesized from ring closure reactions of compound 3 with $\pi$-acceptors activated carbon compounds in different medium and conditions. Structures of the products are characterized by MS, IR, UV-VIS, CH, $\mathrm{N}$, and ${ }^{1} \mathrm{H} /{ }^{13} \mathrm{CNMR}$ spectral data. The new products have been evaluated as potential inhibitors towards HIV-1 activity.
\end{abstract}

Keywords

Synthesis, Fluorine, Phosphorus, Sulfar 1,2,4-Trizinones HIV-1

\section{Introduction}

Organophosphorus systems are ubiquitous in nature and exhibit many applications in the field of agriculture medicine and industry [1] [2]. Many multi-ring phosphorus heterocycles are used as pesticide [3], bactericide

*Corresponding author.

How to cite this paper: Abdel-Rahman, R.M., Makki, M.S.T. and Al-Romaizan, A.N. (2014) Synthesis of Novel Fluorine Substituted Isolated and Fused Heterobicyclic Nitrogen Systems Bearing 6-(2'-Phosphorylanilido)-1,2,4-Triazin-5-One Moiety as Potential Inhibitor towards HIV-1 Activity. International Journal of Organic Chemistry, 4, $247-268$.

http://dx.doi.org/10.4236/ijoc.2014.44028 
[4]-[6], antibiotics [4], and acts as HIV protease inhibitors [7]. Thus, synthesis of new phosphorus bearing a heterocycles has attracted the attention of researchers. Phosphorylation of organic compounds often improves their biological activity, especially through a vital energy, because the P-O is the store of energy for metabolism process. For example, phophorylated- $\mathrm{N}$ in the nucleocapsid affects the interaction between the $\mathrm{N}$-atom and the genomic RNA. The charge repulsion between the negatively charged phosphoserine and the negatively charged RNA may weaken the interaction between $\mathrm{N}$-atom and RNA, thus enabling the viral polymerase to gain access to genomic RNA and to initiate viral RNA transcription and replication [8]. On the other hand, chemistry of N-phosphorylheterocycles showed that these compounds from two dimensional polymeric chains via intermolecular $\mathrm{P}-\mathrm{O}^{-+} \mathrm{H}-\mathrm{N}$ hydrogen bonds [9]. Also, phosphodiester compound had a type of action, especially enzymetic of DNA replication [10] on DNA ligase as (Figure 1).

It is interesting that fluorine containing aheterocycles bearing functional groups exhibits highly effective in biological process, pharmaceuticals, agrochemicals, polymers and a wide range of consumer products [11]. It reflects its resistance to metabolic change due to the strength of the C-F bond providing biological stability and the application of its nonstick-interfacial physical characteristics [12]-[16]. Abdel-Rahman et al. [17]-[21] reported the synthesis and chemical reactivity of 3-thioxo-1,2,4-triazin-5 one derivatives as a bioactive molecules, especially as anti-cancer, anti-AIDS, Amyllolytic, cellobiase and antimicrobial agents. Based upon these observations, the aim of this work is to study the formation of 6-(5'-fluoro-2'-phosphoryl anilido-3-hydrazino-1,2,4triazin-5 $(2 \mathrm{H})$ one then study their behaviour as electron donor towards different electron acceptors such as carbo-sulfur, oxygen, halogen and nitrogen compounds; finally, a type of isolated and/or fused heterobicyclic systems obtained and evaluation as potential inhibitors towards HIV-1 virus.

\section{Experimental}

Melting points were determined with an electro-thermal Bibbly Stuart Scientific Melting point SMPI (UK). A perkin Elmer (Lambda EZ-2101) double beam spectrophotometer $(190$ - $1100 \mathrm{~nm})$ used for recording the electronic spectra. A Perkin Elmer model RXI-FT-IR 55,529 $\mathrm{cm}^{-1}$ used for recording the IR spectra (EtOH as solvents). A Brucker advance DPX $400 \mathrm{MHz}$ using TMS as an internal standard for recording the ${ }^{1} \mathrm{H} /{ }^{13} \mathrm{C}$ NMR spectra in deuterated DMSO ( $\delta$ in pp m). AGC- MS-QP 1000 Ex model is used for recording the mass spectra. Hexafluorobenzene was used as external standard for ${ }^{19} \mathrm{FNMR}$ at $84.25 \mathrm{MHz}$ and ${ }^{31} \mathrm{P}$ (in $\mathrm{CDCl}_{3}, 101.25 \mathrm{MHZ}$ ).

Elemental analysis was performed on Micro analytical Center of National Reaches Center-Dokki, Cairo, Egypt. Compound 1 prepared according the reported method [17].

\section{6-(2'-aminos-5'-fluorophenyl-3-thioxo-1,2,4-triazin-5(2H)one (1)}

A mixture of 5 -fluoroisatin $(0.01 \mathrm{~mol})$ in sodium hydroxide solution $(5 \%, 50 \mathrm{ml})$ warm for $10 \mathrm{~min}$, then thiosemicarbazide $(0.01 \mathrm{~mol}$, in hot water, $10 \mathrm{ml})$ add and complete the refluxing for $2 \mathrm{~h}$. The reaction mixture cooled then poured onto ice and neutralize with diluted $\mathrm{HCl}$. The solid thus obtained filtered off and crystallized from ethanol to give 1 as yellow crystals, yield $80 \%$; m.p. $265^{\circ} \mathrm{C}$. Analytical data; found: C, 44.91; H, 2.90; F, 7.58: N, 23.40; S, 13.29\%.Calculated for $\mathrm{C}_{9} \mathrm{H}_{7} \mathrm{FN}_{4} \mathrm{OS}$ (238); C, 45.37; H, 2.94; F, 7.98; N, 23.52; S, 13.44\%.UV: $\lambda_{\max }(\mathrm{EtOH})=280 \mathrm{~nm} . \mathrm{IR} v \mathrm{~cm}^{-1}: 3424\left(\mathrm{NH}_{2}\right), 3258,3169(\mathrm{NH}, \mathrm{NH}), 1685(\mathrm{C}=\mathrm{O}), 1618\left(\right.$ deform. $\left.\mathrm{NH}_{2}\right), 1545$ $(\mathrm{C}=\mathrm{N}), 1263$ (C-F), 858,818 (aryl CH), 685 (C-F). ${ }^{1} \mathrm{H}$ NMR (DMSO) $\delta=14.66,12.66,10.90$ (each s, 1H, 3NH), 8.68 - 8.06, 7.69 - 7.64, 7.39 - 7.30, (s, 3H, aryl protons). ${ }^{13} \mathrm{C}$ NMR (DMSO): $\delta 179.47(\mathrm{C}=\mathrm{S}), 162$ (C=O), 159 157 (C-F), $138.54(\mathrm{C}=\mathrm{N}), 131.82,121.8,121.51$ (aromatic carbons, 78.14, 77.71 ( $\mathrm{C}_{5}-\mathrm{C}_{6}, 1,2,4$-trinzine). M/Z (Int. \%); 256 (M + $\left.\mathrm{H}_{2} \mathrm{O}, 5 \%\right), 68$ (100), 148(21), 138(18), 110 (30), 96 (50), 82(58.0), 70(78).

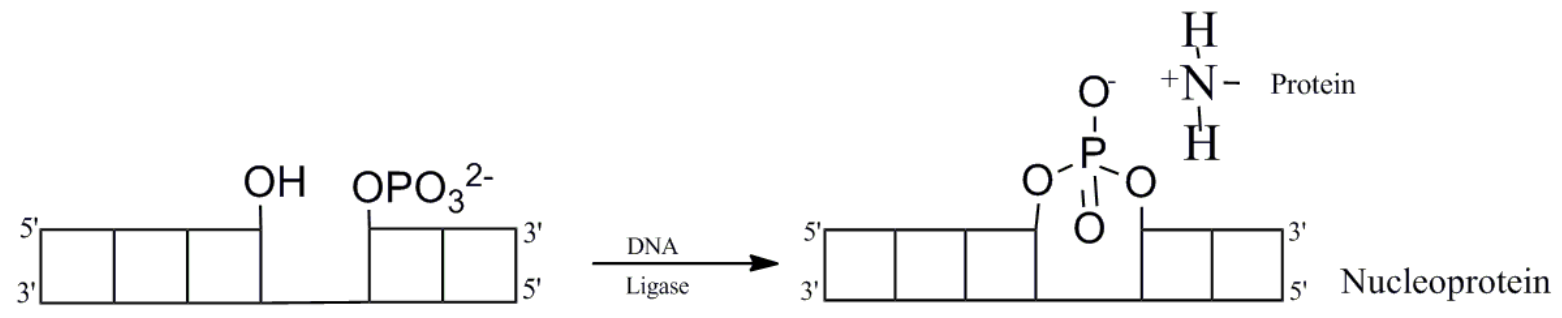

Figure 1. The reaction of DNA ligase phospho diester link. 
6-(5'-Fluoro-2'-diphenylphosphorylanilido)-3-thioxo-1,2,4-triazin-5(2H)one (2)

Equimolar mixture of compound $\mathbf{1}$ and diphenylphosphoryl chloride in DMF (20 ml) warm for $1 \mathrm{~h}$, cooled then poured onto ice. The produce solid filter off and crystallized from methanol to give $\mathbf{2}$ as deep yellow crystals. Yield 70\%; m.p. 218-220 ${ }^{\circ} \mathrm{C}$. Analytical data; found: C, 53.39; H, 3.51; F, 3.88: N, 11.69\%.Calculated for $\mathrm{C}_{21} \mathrm{H}_{16} \mathrm{FN}_{4} \mathrm{PSO}_{4}(470)$; C, 53.61; H, 3.70; F, 4.04; N, 11.91\%. IR $v \mathrm{~cm}^{-1}: 3133.8(\mathrm{NH}), 1688.4(\mathrm{C}=\mathrm{O}), 1574$ (C=N), 1370 (Cyclic NCSN), 1262 (C-F), 1200 (P=O), 1150 (C-S), 10.96 (Ph-O-P), 858, 801 (substituted phenyls) M/Z: (Int.\%); 473 (M + 3, 5.11), 110 (100).

3-Hydrazino-6-(5'-fluoro-2'-diphenylphosphorylanilido)-1,2,4-triazin-5(2H)one (3)

A mixture of compound 2 (1 gm) and hydrazine hydrate $(2 \mathrm{ml})$ in ethanol $(50 \mathrm{ml})$ reflux for $2 \mathrm{~h}$. cooled. The solid thus produce filter off and crystallized from ethanol to give 3 as orange crystals, yield $85 \%$; m.p. $178^{\circ} \mathrm{C}$ $180^{\circ} \mathrm{C}$. Analytical data; found: C, 53.45; $\mathrm{H}, 3.59 ; \mathrm{F}, 3.80 \%$.Calculated for $\mathrm{C}_{21} \mathrm{H}_{18} \mathrm{FN}_{6} \mathrm{PO}_{4}(468)$, C, 53.84; $\mathrm{H}$, 3.84; F, 4.02; N, 17.14\%. IR $v \mathrm{~cm}^{-1}=3381,3340,3220-3190\left(\mathrm{NH}, \mathrm{NH}, \mathrm{NH}_{2}\right), 1682(\mathrm{C}=\mathrm{O}), 1558(\mathrm{C}=\mathrm{N})$, 1266.9 (C-F), 1210-1156 (P=O). 1050 (Ph-O-P), 860,805 (substituted phenyls). ${ }^{1} \mathrm{H}$ NMR(DMSO) $\delta=10.7,10.2$, 8.7 (each s, 3H, 3NH, 1,2,4-triazino NH-P=O), 7.511, 7.27-2.24, 7.23-7.22 (each s, 3H, aryl protons); 7.143 7.002; 6.860 - 6.796 (each m, $10 \mathrm{H}$, phenyl protons), 2.88 (s, $2 \mathrm{H}, \mathrm{NH}_{2}$ of hydrazine), ${ }^{13} \mathrm{C}$ NMR (DMSO) $\delta=$ 163.78, 159.44, 157.86, 134.97, 129.07, 127.65, 123.66, 122.87, 120.12, 120.09, 113.47, 113.31, 110.77, 110.71, 107.93, 105.27, 105.10, 77.76, 77.33.

3-(3'-Amino-4'-carboxy-5-(4-chlorophenyl)-4',5'-dihydro-pyrazolin-1'-yl)-6-(5'-fluoro-2'-diphenylphosphorylanilido)-1,2,4-triazin-5(2H)one (4)

Equimolar amounts of $\mathbf{3}$ and $\alpha$-(4-chlorophenylidene) cyano acetic acid in ethanol (100 $\mathrm{ml}$ ) and a few drops piperidine $(0.5 \mathrm{ml})$ reflux for $8 \mathrm{~h}$, cooled, then added off and crystallized from dioxan to give $\mathbf{4}$ as yellowish crystals, yield $65 \%$ m.p., $158^{\circ} \mathrm{C}-160^{\circ} \mathrm{C}$. Analytical data; found: C, 54.81; H, 3.11; F, 2.55; Cl, 5.09; N, $14.29 \%$. Calculated for $\mathrm{C}_{31} \mathrm{H}_{24} \mathrm{FClN}_{7} \mathrm{PO}_{6}(675)$. C, 55.11; H, 3.55; F, 2.81; Cl, 5.18; N, 14.51\%; M/S: 675 (1.11), 95(100). IR $v \mathrm{~cm}^{-1}$ : $3420(\mathrm{OH}), 3381,3155\left(\mathrm{NH}, \mathrm{NH}_{2}\right), 1680(\mathrm{C}=\mathrm{O})$ 1558(C=N), 1478 (deform aliphatic CH), 1269 (C-F), 1200 (P=O), 1043 (Ph-O-P), 993.861, 804 (Aryl CH). ${ }^{1} \mathrm{H}$ NMR (DMSO) $\delta=10.67,10.14$, 8.72 (each $\delta$, 3H, 3NH, 1,2,4-triazinare NH-P=O), $9.77(\delta, 1 \mathrm{H}, \mathrm{OH}), 8.15$ - 7.52, 7.36 - 7.29, 7.13 - 7.00, 6.991 - 6.811 (each m, $19 \mathrm{H}, 7 \mathrm{H}$ aryl, $10 \mathrm{H}$ phenyls pyrazole protons); $3.46\left(\delta\right.$, $\left.2 \mathrm{H}, \mathrm{NH}_{2}\right){ }^{13} \mathrm{C}$ NMR (DMSO) $\delta$ : $179.64 ; 163.76 ; 163.05$; 159.42; 157.84; 138.58; 134.94; 128.65; $127.59 ; 123.72 ; 123.66 ; 121.13 ; 121.07 ; 117.67 ; 117.51 ; 113.45$; 113.28; 112.06; 112.01; 110.75; 110.70; 109.66; 108.09; 107.92; 105.25; 105.08; 77.78; 77.35; 23.64.

3-(3'-Methyl-5'-arylamino-pyrazolin-1'-yl)-6(5'-fluoro-2'-diphenylphosphorylanilido)-1,2,4-trinzin-5(2 H) one (5)

A mixture of $3(0.01 \mathrm{~mol})$ and acetyl acetanilide derivative $(0.01 \mathrm{~mol})$ in DMF $(50 \mathrm{ml})$ reflux for $2 \mathrm{~h}$, cooled then poured onto ice. The solid thus yield filtere off and crystallized from dioxan to give $\mathbf{5}$ as deep-yellowish crystals, yield $70 \%$; m.p. $100^{\circ} \mathrm{C}-101^{\circ} \mathrm{C}$. Analytical data; found C, 56.03; H, 3.41; F, 2.23; N, 16.17; S, 3.91\%. Calculated for $\mathrm{C}_{36} \mathrm{H}_{29} \mathrm{FN}_{9} \mathrm{PSO}_{6}(765)$. C, 56.47; H, 3.79; F, 2.48; N, 16.47; S, 4.18\%. IR $v \mathrm{~cm}^{-1}: 3424,3220$ $31.70(\mathrm{NH}, \mathrm{NH}), 2932\left(\mathrm{CH}_{3}\right), 1694(\mathrm{C}=\mathrm{O}), 1620(\mathrm{C}=\mathrm{N}), 1358\left(\mathrm{SO}_{2}-\mathrm{NH}\right), 1268(\mathrm{C}-\mathrm{F}), 1220(\mathrm{P}=\mathrm{O}), 1054$ (Ph-O-P), 952, 909, 810, 758 (aryl CH). ${ }^{1} \mathrm{H}$ NMR (DMSO) $\delta=12.28,12.27,10.36,10.21$ (each s, 4H, 4NH) 9.7 (s, $1 \mathrm{H}, \mathrm{C}_{4^{-}}$of pyrazole), 7.979, 7.971, 7.556, 7.545 (m, 4- $\mathrm{H}$ of pyridine); 7.34, 7.336, 7.322, 7.091(m, $4 \mathrm{H}$ of aryl-P-SO $\mathrm{SO}_{2} \mathrm{NH}$ ) 6.95 - 6.83; 6.66 - 6.52; 6.41 - 6.16 (each m, $13 \mathrm{H}$ of $\mathrm{FC}_{6} \mathrm{H}_{3}, 2 \mathrm{C}_{6} \mathrm{H}_{5}$ ). 0.46 - 0.45 (s, $3 \mathrm{H}, \mathrm{CH}_{3}$ ). ${ }^{13} \mathrm{C}$ NMR(DMSO) $\delta=179.64,163.75,163.03,162.41,158.08,157.99,157.47 ; 151.97 ; 138.58,134.94,130.23$, 129.10, 123.72, 123.66, 117.66, 117.50, 115.11, 113.41, 113.25, 112.97, 112.07, 112.02, 110.76, 110.71, 108.09, 107.92, 105.21, 105.04, 77.83-77.40, 36.40, 31.29.

3-(3'-Amino-4',5'-dihydro-5'-oxo-pyrazolin-1'-yl)-6(5'-fluoro-2'-diphenylphosphorylanilido)-1,2,4-triazin5(2H)one (6)

Equiomolar of 3 and ethyl cyanoacetate in THF $(100 \mathrm{ml})$ reflux for $4 \mathrm{H}$, cooled. The solid thus produce filtered off and crystallized from THF to give 6 as faint yellow crystals, yield 68\%; m.p. 200-202 ${ }^{\circ} \mathrm{C}$. Analytical data; found: C, 53.53; H, 3.31; F, 3.29; N, 18.01\%. Calculated for $\mathrm{C}_{24} \mathrm{H}_{19} \mathrm{FN}_{7} \mathrm{PO}_{5}(535)$; C, 53.83; H, 3.55; F, 3.55; N, 18.31\%. IR $v \mathrm{~cm}^{-1}$ : $3425\left(\mathrm{NH}_{2}\right), 3220-3190(\mathrm{NH} \rightleftarrows \mathrm{OH}), 1694(\mathrm{C}=\mathrm{O}), 1471$ (deformation. $\left.\mathrm{CH}_{2}\right), 1310(\mathrm{~N}-\mathrm{N})$, 1250 (C-F), 1210 (P=O), 1058 (Ph-O-P), 952, 915, 808 (aryl CH). ${ }^{1} \mathrm{H}$ NMR (DMSO) $\delta:$ 12.7, 10.67, 8.8 (each s, $3 \mathrm{H}, 3 \mathrm{NH}$ ), 10.24 (s, 1H, OH of pyrazole), 7.66 - 7.400, 7.39-7.00, 6.99-6.79 (each m, 14H, aryl \& phenyl protons). ${ }^{13} \mathrm{C}$ NMR(DMSO) $\delta: 179.54,163.70,159.31,157.74,134.91,128.98,127.31,123.68,117.57,113.29$, 113.13, 111.95, 110.69, 110.64, 108.23, 105.08, 104.91, 78.0-77.54.

3-(3',5'-Diaminopyrazolin-1'-yl)-6-(5'-fluoro-2'-diphenylphosphorylanilido)-1,2,4-triazin-5(2H)one (7) 
A mixture of $3(0.01 \mathrm{~mol})$ and malononitrile $(0.01 \mathrm{~mol})$ in ethanol $(50 \mathrm{ml})$ and piperidine $(0.5 \mathrm{ml})$ reflux for $8 \mathrm{~h}$, cooled. The solid obtained filtered off and crystallized from ethanol to give 7 as deep orange crystals. Yield $70 \%$; m.p. $205^{\circ} \mathrm{C}-207^{\circ} \mathrm{C}$. Analytical data; found: C, 53.55, H, 3.12; F, 3.31; N, 20.71\%. Calculated for $\mathrm{C}_{24} \mathrm{H}_{21} \mathrm{FN}_{8} \mathrm{PO}_{4}$ (535); C, 53.83; H, 3.55; F, 3.55; N, 20.93\%. IR: $v \mathrm{~cm}^{-1} 3430-3380\left(\mathrm{NH}_{2}\right), 1700(\mathrm{C}=\mathrm{O}), 1620$ (deform. $\mathrm{NH}_{2}$ ), 1580 (C=N) 1265 (C-F), 1200 (Ph-P=O), 1050 (Ph-O-P), 930, 910, 850, 800(aryl CH). ${ }^{1} \mathrm{H}$ NMR (DMSO) $\delta$ : 12.7, 10.7, 10.2 (each s, 3H, 3NH), 8.8 (s, 1H, NH-P=O), 7.55 - 7.311, 7.131 - 6.991, 6.87 - 6.76 (each m, $14 \mathrm{H}$, aryl \& phenyl protons), $3.89\left(\mathrm{~s}, 4 \mathrm{H}, 2 \mathrm{NH}_{2}\right) .{ }^{13} \mathrm{C}$ NMR (DMSO) $\delta: 163.73,159.38,157.80,134.93$, 127.49, 123.67, 113.38, 113.22, 110.66, 105.18; 105.01, 77.84-77.41, M/S (Int.\%): 534 (536, M + 2, 1.55\%), 248 (1.11), 97 (3.18) 96 (5.55), 95 (100), 93 (18.11), 68 (42.00), 67 (3.11), 62 (37.15).

3-(5'-Phenyl-3'-oxo-2,3,4,5-tetrahydro-pyrazolin-1'-yl)-6-(5'-fluoro-2'-diphenyl phosphorylanilido)-1,2, 4-triazin-5-(2H)one(8)

Equimolar amounts of 3 and cinnamoyl chloride in DMF (20ml) reflux for $4 \mathrm{~h}$, cooled then poured onto ice. The produce solid filtered off and crystallized from dioxan to give $\mathbf{8}$ as deep yellowish crystals, yield $60 \%$, m.p. $160^{\circ} \mathrm{C}$. $-162^{\circ} \mathrm{C}$. Analytical data; found: C, 59.89; H, 3.55; F, 3.01; N, $13.75 \%$. Calculated for $\mathrm{C}_{30} \mathrm{H}_{23} \mathrm{FN}_{6} \mathrm{PO}_{5}$ (597); C, 60.30; H, 3.85; F, 3.18; N, 14.07\%. IR $v \mathrm{~cm}^{-1}$ : $3428(\mathrm{OH}), 3180(\mathrm{NH}), 2937\left(\mathrm{CH}_{2}\right), 1694(\mathrm{C}=0), 1610$ $(\mathrm{C}=\mathrm{N}), 1482$ (deform. $\mathrm{CH}_{2}$ ), 1314 (N-N), 1230 (C-F), 1169 (P=O), 1059 (Ph-O-P), 954, 909, 809 (aryl CH). ${ }^{1} \mathrm{H}$ NMR (DMSO) $\delta: 14.7,13.5,12.6$ (each s, 3H, 3NH), 10.80 (s, 1H, OH of pyrazole), 8.8 (s, 1H, NH-P=O), 7.99 - 7.41, 7.04 - 7.32, 7.10 - 6.93, 6.88 - 6.40 (each m, $20 \mathrm{H}$, aryl and phenyl protons), $3.23\left(\mathrm{~s}, 2 \mathrm{H}, \mathrm{NH}_{2}\right) .{ }^{13} \mathrm{C}$ NMR (DMSO) $\delta$ : 163.70, 163.58, 162.90, 162.24, 159.34, 157.83, 157.76, 144.07, 134.29, 130.01, 128.77, 127.84, 121.16, 118.94, 118.84, 117.43, 114.23, 113.13, 112.97, 111.94, 111.89, 111.48, 111.43, 110.64, 110.59, 108.30, 108.12, $78.13-77.91,36.26,31.14$.

3-(3'-(4''-Nitrophenyl)-5'-(4'’-fluorophenyl)-4'-5'-dihydro-pyrazolin-1'-yl)-6-(5'-fluoro-2'-diphenyl-phos-phorylanilido)-1,2,4-triazin-5(2H)one (9)

A mixture of $3(0.01 \mathrm{~mol})$ and a chalcone $(0.01 \mathrm{~mol})$ in ethanol $(50 \mathrm{ml})$, and piperidine $(0.5 \mathrm{ml})$ reflux for $8 \mathrm{~h}$, cooled then poured on ice-HCl. The solid produce filtered off and crystallized from THF to give $\mathbf{9}$ as yellow crystals, yield $82 \%$, m.p. $148^{\circ} \mathrm{C}-150^{\circ} \mathrm{C}$. Analytical data; found: C, 59.49; H, 3.51; F, 5.21; N, $13.29 \%$. Calculated for $\mathrm{C}_{36} \mathrm{H}_{26} \mathrm{~F}_{2} \mathrm{~N}_{7} \mathrm{PO}_{6}$ (721); C, 59.91; H, 3.60; F, 5.27; N, 13.59\%. IR $v \mathrm{~cm}^{-1}: 3180(\mathrm{NH}), 2827\left(\mathrm{CH}_{2}\right), 1675$ $(\mathrm{C}=\mathrm{O}), 1595(\mathrm{C}=\mathrm{N}), 1547(\mathrm{C}=\mathrm{N})$, 1500, 1423 (deform. $\mathrm{CH}_{2}$ ), 1291 (C-F), 1225 (Ph-P=O), 1097 (Ph-O-P), 921, 847, 762 (aryl CH), $684(\mathrm{C}-\mathrm{Cl}) .{ }^{1} \mathrm{H}$ NMR (DMSO) $\delta=10.57,10.115$ (each s, 2H, 2NH), $8.160-8.157$, (s, 1H, NH-P=O), 8.118 - 8.095, 7.838 - 7.49, 7.23 - 7.010, 6.92 - 6.90, 6.73 - 6.70 (each m, 23H, aryl \& phenyl protons) $2.49-2.48\left(\delta, 2 \mathrm{H}_{,} \mathrm{CH}_{2}\right) .{ }^{13} \mathrm{C}$ NMR (DMSO) $\delta=163.72,159.35,157.78,134.92,130.85,130.80,129.46,127.41$, 123.86, 123.73, 123.67, 121.10, 113.34, 113.18, 111.98, 110.72, 110.67, 108.09, 107.92, 105.13, 104.97, 77.92 77.50, 39.56 .

3-(3',5'-Dioxo-2',3',4',5'-tetrahydro-pyrazolin-1'-yl)-6-(5'-fluoro-2'-diphenyl phosphorylanilido)-1,2,4triazin-5'(2H)one (10)

Equimolar mixture of 3 and diethyl malonate in THF $(100 \mathrm{ml})$ reflux for $8 \mathrm{~h}$, cooled. The solid thus obtain filtered off and crystallized from dioxan to give $\mathbf{1 0}$ as faint yellow crystals, yield $66 \%$, m.p. $189-190^{\circ} \mathrm{C}$. Analytical data; found: C, 53.35; $\mathrm{H}, 3.18$; F, 3.35; N, 15.38\%. Calculated for $\mathrm{C}_{24} \mathrm{H}_{18} \mathrm{FN}_{6} \mathrm{PO}_{6}(536)$; C, 53.73; $\mathrm{H}, 3.35 ; \mathrm{F}$, 3.54; N, 15.67\%. IR $v \mathrm{~cm}^{-1}$ : $3300(\mathrm{NH}), 3169(\mathrm{NH}), 1694(\mathrm{C}=\mathrm{O}), 1626(\mathrm{C}=\mathrm{N}), 1579(\mathrm{C}=\mathrm{N}), 1471$ (deform. $\left.\mathrm{CH}_{2}\right)$, 1304 (N-N), 1265 (C-F), 1196 (P=O), 1040 (Ph-O-P), 903, 863, 809 (aryl CH). ${ }^{1} \mathrm{H}$ NMR (DMSO) $\delta=12.7$, 10.69, 10.35 (each s, 3H, NH, OH, OH) 9.21 (s, 1H, NH-P=O), 8.15 (s, $1 \mathrm{H}, \mathrm{C}_{4}$ of pyrazole), 7.79 - 7.38, 7.10 7.003, 6.99 - 6.79 (each m, 15H, aryl \& phenyl protons), $4.18-4.17,\left(\delta, \mathrm{CH}_{2}\right.$ of pyrazole). ${ }^{13} \mathrm{CNMR}$ (DMSO) $\delta=$ 179.39, 163.58, 162.91, 159.18, 157.61, 134.86, 126.95, 123.74, 123.68, 117.44, 117.28, 113.13, 112.97, 111.94, 110.64, 110.58, 108.12, 107.95, 104.89, 104.72, 78.29 - 77.85, 61.09, 13.94. M/S (Int.\%): 536 (538, M + 2, 2.28), 99 (5.5), 96 (13.11), 95 (100), 93 (36.11), 69 (21.85), 68(42.35), 62(5.18).

3-(5'-Aryl-3'-thioxo-2',3'-dihydro-1',2',4'-triazol-1'-yl)-6-(5'-fluoro-2'-diphenylphosphorylanilido)-1,2, 4-triazin-5(2H) one (11)

A mixture of $3(0.01 \mathrm{~mol})$ and P-methoxybenzoylisothiocyanate $(0.01 \mathrm{~mol})$ in dioxan $(20 \mathrm{ml})$ reflux for $4 \mathrm{~h}$, cooled. The solid produce filtered and crystallized from dioxan to give $\mathbf{1 1}$ as orange yellowish crystals. Yield $65 \%$, m.p. $141^{\circ} \mathrm{C}-192^{\circ} \mathrm{C}$. Analytical data, found: C, 55.62; H, 3.41; F, 2.74; N, 15.02; S, 4.72\%. Calculated for $\mathrm{C}_{30} \mathrm{H}_{23} \mathrm{FNPSO}_{5}$ (643); C, 55.98; H, 3.57; F, 2.95; N, 15.24; S, 4.97\%. IR $v \mathrm{~cm}^{-1}=3220-3180$ (b, NH), 1693 $(\mathrm{C}=\mathrm{O}), 1624,1537$ (C=N), 1477 (deform. MeO), 1305(N-N), 1266(C-F), 1155 (C-S), 1099 (P=O), 1039(P-O), 980, 840, 809 (aryl CH). ${ }^{1} \mathrm{H}$ NMR(DMSO) $\delta=12.74,10.80$ - 1066, 8.73 (each s, 3H, 3NH), 8.40 - 7.52, 7.38 - 
7.30, 7.29 - 7.00, 6.99 - 6.800 (each m, 17H) aryl \& phenyl protons), 3.68 - 3.67 (s, 3H, OMe). ${ }^{13} \mathrm{C}$ NMR(DMSO) $\delta: 179.64,163.77,163.04,159.40,158.02,157.85,138.58,134.94,132.10,127.60,123.72$, 123.66, 121.13, 121.07, 117.69, 117.53, 113.46, 113.28, 112.02, 110.76, 110.71, 108.10, 107.93, 105.25, 105.07, 77.81 - 77.35, 66.91.

3-(3'-(4'-Methoxyphenyl)-5'-thioxo-4',5'-dihydro-1'2',4'-triazol-1'-yl)-6-(5'-fluoro-2'-diphenylphos phorylanilido)-1,2,4-triazin-5(2H)one (12)

A mixture of 3 (0.01 mol) and 4-methoxybenzoyl isothiocynate $(0.01 \mathrm{~mol})$ is DMF $(20 \mathrm{ml})$ reflux for $4 \mathrm{~h}$, cooled then poured onto ice. The produce solid filtered off and crystallized from ethanol (to give $\mathbf{1 2}$ as orange crystals, yield $70 \%$, m.p. $186^{\circ} \mathrm{C}-188^{\circ} \mathrm{C}$. Analytical data: found: C, 55.69; H, 3.55; F, 2.70; N, 14.89; S, $4.79 \%$. Calculated for $\mathrm{C}_{30} \mathrm{H}_{23} \mathrm{FN}_{7} \mathrm{PSO}_{5}$ (643); C, 55.98; H, 3.57; F, 2.95; N, 15.24; S, 4.97\%. IR $v \mathrm{~cm}^{-1}$ : $3382(\mathrm{NH})$, $3318(\mathrm{NH}), 3204(\mathrm{NH}), 1684(\mathrm{C}=\mathrm{O}), 1562(\mathrm{C}=\mathrm{N}), 1484$ (deform. MeO), 1311 (N-N), 1270 (C-F), 1169 (C=S), 1041 (Ph-O-P), 995, 881, 807 (aryl CH).

3-Methyl-1H-7-(5'-fluoro-2'-diphenyl phosphorylanilido)-1,2,4-triazino[4,3-b][1,2,4] triazin-4,8-dione (13)

A mixture of $3(0.01 \mathrm{~mol})$ and sodium pyruvate $(0.01 \mathrm{~mol})$ in sodium hydroxide solution $(5 \%, 100 \mathrm{ml})$ warm under reflux for $2 \mathrm{~h}$, cooled them poured onto ice $\mathrm{HCl}$. The solid thus obtained filtered off and crystallized $70 \%$, m.p. $220^{\circ} \mathrm{C}-222^{\circ} \mathrm{C}$. Analytical data; found: C, 55.19; H, 3.31; F, 3.38; N, $15.88 \%$. Calculated for $\mathrm{C}_{24} \mathrm{H}_{18} \mathrm{FN}_{6} \mathrm{PO}_{5}$ (520); C, 55.38; H, 3.46; F, 3.65; N, 15\%. IR $v \mathrm{~cm}^{-1}$ : 3380, $3162(\mathrm{NH}, \mathrm{NH}) 1683(\mathrm{C}=\mathrm{O}), 1589,1553(\mathrm{C}=\mathrm{N})$, 1458 (deform. $\mathrm{CH}_{3}$ ), 1308 (N-N), 1250 (C-F), 1204 (P=O), 1040 (Ph-O-P), 909, 861, 804 (aryl CH). ${ }^{1} \mathrm{H}$ NMR (DMSO) $\delta$ : 14.55, 12.8 (each s, 2H, 2NH), 10.06 (s, 1H, NH), 7.66 - 7.40, 7.35 - 7.26, 6.93 - 6.76 (each m, 13H, aryl \& phenyl protons), 5.58 (s, $1 \mathrm{H}, \mathrm{OH}), 1.256\left(\mathrm{~s}, 3 \mathrm{H}, \mathrm{CH}_{3}\right) .{ }^{13} \mathrm{C}$ NMR(DMSO) $\delta: 177.40,176.13,158.14$, 139.40, 128.24, 119.11, 118.95, 118.82, 117.83, 114.59, 114.43, 114.35, 114.29, 112.36, 112.19, 112.09, 112.04, 111.84, 111.77, 111.60, 110.87, 109.95, 109.90, 108.90, 108.37, 77.67 - 77.24, 55.38, 17.21.

\section{Indolo[3,4-e][1,2,4] triazino [4,3-b]-1,2,4-triazin-11-one (14)}

Equimolar amounts of 3 and isatin in DMF $(20 \mathrm{ml})$ reflux for $2 \mathrm{~h}$, cooled then poured onto ice. The yielded solid filtered off and crystallized from dioxan to give $\mathbf{1 4}$ as deep-brown crystals, yield $80 \%$, m.p. $198-200^{\circ} \mathrm{C}$. Analytical data found: C, 59.89; H, 3.01; F, 2.98; N, 16.59\%. Calculated for $\mathrm{C}_{29} \mathrm{H}_{19} \mathrm{FN}_{7} \mathrm{PO}_{4}$ (579); C, 60.10; $\mathrm{H}$, 3.28; F, 3.28, N, 16.92\%. IR $v \mathrm{~cm}^{-1}$ : $3139(\mathrm{NH}), 1694(\mathrm{C}=\mathrm{O}), 1626,1540(\mathrm{C}=\mathrm{N}), 1305(\mathrm{~N}-\mathrm{N}), 1250$ (C-F), 1157 $(\mathrm{P}=\mathrm{O}), 1080$ (Ph-O-P), 970, 900, 850, 811 (aryl CH). ${ }^{1} \mathrm{H}$ NMR(DMSO) $\delta=10.76-10.61$ (s, $\left.1 \mathrm{H}, \mathrm{NH}\right), 8.011$ (s, $1 \mathrm{H}$, NH-P=O), $7.67-7.28,7.27$ - 7.12, $7.05-6.94,6.87-6.816$ (each m, 17H, aryl and phenyl protons). ${ }^{13} \mathrm{CNMR}$ (DMSO) $\delta$ : 163.77, 163.68, 162.45, 159.48, 157.32, 141.29, 136.82, 134.95, 95.133.65, 130.07, 122.27, 120.80, 120.30, 117.73, 117.57, 113.54, 113.50, 113.38, 112.10, 112.04, 110.79, 110.73, 108.09, 107.92, 105.34, 105.17, 77.67 - 77.24. M/Z (Int. \%) 579 (581, M + 2; 13.15\%) 331 (31.88), 275 (75.19), 248 (1.15); 141 (18.20), 95 (100), 93 (20.51), 63 (5.13).

3-Phenyl-1,2,3,4-tetrahydro-7-(5'-fluoro-2'-diphenyl phosphorylanilido)-1,2,4-triazino [4,3-b] [1,2,4] triazin8-one (15)

A mixture of $3(0.01 \mathrm{~mol})$ and phenacyl bromide $(0.01 \mathrm{~mol})$ in ethanolic $\mathrm{KOH}(5 \%, 20 \mathrm{ml})$ reflux for $2 \mathrm{~h}$, then poured onto ice-HCl. The solid produced filtered off and crystallized from THF to give $\mathbf{1 5}$ as brownish crystals. Yield 65\% m.p., $98^{\circ} \mathrm{C}-100^{\circ} \mathrm{C}$. Analytical data; Found: C, 59.98; H, 3.55; F, 3.20; N, 14.51\%. Calculated for $\mathrm{C}_{29-}$ $\mathrm{H}_{22} \mathrm{FN}_{6} \mathrm{PO}_{4}$ (568); C, 61.26; H, 3.87; F, 3.34; N, 14.78\%. IR $v \mathrm{~cm}^{-1}$ : 3158 (NH), 1655 (C=O), 1580 (C=N), 1474 (deform. $\mathrm{CH}_{2}$ ), $1308(\mathrm{~N}-\mathrm{N}), 1230(\mathrm{C}-\mathrm{F}), 1180(\mathrm{P}=\mathrm{O}), 1092$ (Ph-O-P), 980, 950, 900, 850, 801 (aryl $\left.\mathrm{CH}\right){ }^{1} \mathrm{H}$ NMR(DMSO) $\delta=12.75$ (s, 1H, NH), 10.78 (s, 1H, NH-P=O), 7.84 - 7.55, 7.49 - 7.30, 7.29 - 7.00, 6.99 - 6.79 (each m, 18H, aryl \& phenyl protons). $3.4\left(\mathrm{~s}, 2 \mathrm{H}, \mathrm{CH}_{2}\right) .{ }^{13} \mathrm{C}$ NMR (DMSO) $\delta: 179.66,163.04,159.62,158.02$, 138.60, 129.51, 129.14, 128.67, 128.45, 128.18, 127.18, 126.89, 126.67, 125.86, 125.44, 123.31, 121.05, 120.20, 117.72, 117.56, 113.90, 113.74, 112.21, 112.13, 112.07, 110.47, 110.04, 108.10, 107.92, 77.72-77.29, 36.62.

7-(5'-Fluoro-2'-diphenylphophorylanilido)-1,2,3,4-tetrahydro-1,2,4-triazino[4,3-b][1,2,4]triazin-4,8-dione (16)

A mixture of $3(0.01 \mathrm{~mol})$ and monochloroacetic acid $(0.01 \mathrm{~mol})$ in DMF $(20 \mathrm{ml})$ reflux for $2 \mathrm{~h}$, cooled then poured onto ice. The solid thus obtained filtered off and crystallized from THF to give $\mathbf{1 6}$ as yellowish crystals. Yield $70 \%$, m.p. $165^{\circ} \mathrm{C}-167^{\circ} \mathrm{C}$. Analytical data; found: C, 54.01; H, 3.25; F, 3.58; N, $16.31 \%$. Calculated for $\mathrm{C}_{23} \mathrm{H}_{18} \mathrm{FN}_{6} \mathrm{PO}_{5}$ (508); C, 54.33; H, 3.54; F, 3.74; N, 16.53\%. IR $v \mathrm{~cm}^{-1}$ : $3382(\mathrm{NH}), 3224(\mathrm{NH}), 1685(\mathrm{C}=\mathrm{O})$, $1590(\mathrm{C}=\mathrm{N}), 1477$ (deform. $\mathrm{CH}_{2}$ ), 1250 (C-F), $1180(\mathrm{P}=\mathrm{O}), 1041$ (Ph-O-P), 807, 760 (aryl CH). ${ }^{1} \mathrm{H}$ NMR (DMSO) $\delta$ : 12.97, 10.70, 10.42 (each s, 3H, 2NH, OH), 9.19 (s, 1H, NH-P=O), 7.99 - 7.00, 6.99 - 6.86, 6.85 6.79 (each $\mathrm{m}, 13 \mathrm{H}$, aryl \& phenyl protons), 4.33 (s, $2 \mathrm{H}, \mathrm{CH}_{2}$ ). ${ }^{13} \mathrm{C}$ NMR (DMSO) $\delta=173.41,163.77,163.67$, 
159.42, 157.84, 157.70, 136.69, 136.04, 121.23, 121.06, 113.87, 113.81, 113.24, 113.08, 111.48, 111.43, 110.966, 110.699, 111.644, 109.28, 107.00, 106.83, 105.02, 104.02, 78.08 - 77.65, 31.22.

7-(5'-Fluoro-2'-diphenylphosphorylanilido)-1,2-dihydro-1,2,4-triazino $[4,3-b][1,2,4]$ triazin-3,8-dione (17)

Equimolar mixture of 3 and 1,1-dichloroacetic acid in DMF (20ml) reflux for $2 \mathrm{~h}$, cooled then poured onto ice. The yield obtained filtered and crystallized from dioxan to 17 as yellowish crystals. Yield $78 \%$, m.p. $180^{\circ} \mathrm{C}-$ $182^{\circ} \mathrm{C}$. Analytical data: found C, 54.03; H, 2.89; F, 3.55; N, 16.31\%. Calculated for $\mathrm{C}_{23} \mathrm{H}_{16} \mathrm{FN}_{6} \mathrm{PO}_{5}(506) ; \mathrm{C}$, 54.54; H, 3.16; F, 3.75; N, 16.60\%. IR $v \mathrm{~cm}^{-1}$ : $3100(\mathrm{NH}), 1791(\mathrm{C}=\mathrm{O}), 1655(\mathrm{C}=\mathrm{O}), 1590(\mathrm{C}=\mathrm{N}), 1547(\mathrm{C}=\mathrm{N})$, 1295 (N-N), 1235 (C-F), 1158 (P=O), 1094 (Ph-O-P), 984, 865, 830 (aryl CH). ${ }^{1} \mathrm{H}$ NMR (DMSO) $\delta: 12.7$ (s, $1 \mathrm{H}$, NH), 10.53 (s, 1H, NH-P=O), 9.07 (s, 1H, CH=N), 7.91 - 7.16, 6.92 - 6.81, 6.77 - 6.71 (each m, 13H, aryl \& aryl protons). ${ }^{13} \mathrm{C}$ NMR(DMSO) $\delta: 179.58,163.82,163.01,162.39,159.50,157.92,142.32,138.56,136.67,136.03$, $121.28,121.12,117.45,115.59,115.43,113.90,112.13,111.47,110.67,109.50,109.34,108.08,107.91,107.12$, 106.95, $77.88-77.45,66.36$.

7-(5'-Fluoro-2'-diphenylphosphorylanilido)-1,2,3,4-tetrahydro-1,2,4-triazino[4,3-b][4,34]triazin-3,4,8trione (18)

A mixture of $3(0.01 \mathrm{~mol})$ in dry $\mathrm{C}_{6} \mathrm{H}_{6}$ treat with oxalyl chloride $(0.01 \mathrm{~mol})$ added dropwise then, TEA added drop wise (few drops) then heated under reflux for $2 \mathrm{~h}$, cooled. The solid produce filtered off and crystallized from $\mathrm{C}_{6} \mathrm{H}_{6}$ to give 18 as deep-yellowish crystals, yields $60 \%$, m.p. $168^{\circ} \mathrm{C}-170^{\circ} \mathrm{C}$. Analytical data: found: C, 52.49; H, 2.85; F, 3.51; N, 15.88\%. Calculated for $\mathrm{C}_{23} \mathrm{H}_{16} \mathrm{FN}_{6} \mathrm{PO}_{6}$ (522); C, 52.87; H, 3.06; F, 3.63; N, $16.09 \%$. IR $v \mathrm{~cm}^{-1}$ : 3382, 3319, $3156(3 \mathrm{NH}), 1680(\mathrm{C}=\mathrm{O}), 1557(\mathrm{C}=\mathrm{N}), 1270(\mathrm{C}-\mathrm{F}), 1143(\mathrm{P}=\mathrm{O}), 1041(\mathrm{Ph}-\mathrm{O}-\mathrm{P}), 994$, 860, 804 (aryl CH). ${ }^{1} \mathrm{H}$ NMR (DMSO) $\delta: 14$ (s, $\left.1 \mathrm{H}, \mathrm{NH}\right), 12.9$ (s, 1H, NH), 10.8 (s, 1H, NH-P=O) 9.3, 8.50, 8.0 (each s, aryl protons), 7.45, 7.35, 7.039, 7.035, 7.031, 7.02, 6.886, 6.878, 6.870, 6.86 (m, 10H phenyl protons). ${ }^{13} \mathrm{C}$ NMR (DMSO) $\delta$ : 167.31, 163.07, 159.53, 158.95, 129.48, 128.26, 120.21, 118.50, 118.26, 112.38, 112.33, 112.19, 112.14, 77.62 - 77.202. M/Z (Int.\%) 522 (525, M + 3, 18.55), 248 (3.15), 96 (23.11), 95 (100), 93 (8.9), 68 (15.0).

The acid hydrazido derivative 20

A mixture of $3(0.01 \mathrm{~mol})$ and oxazolone19 $(0.01 \mathrm{~mol})$ in ethanol $(50 \mathrm{ml})$ with $\mathrm{H}_{2} \mathrm{O}(20 \mathrm{ml})$ reflux for $2 \mathrm{~h}$, cooled then poured onto ice. The produced solid filtered off and crystallized from dioxan to give 20 as yellow crystals. Yield 58\%, m.p. 155-157 ${ }^{\circ} \mathrm{C}$. Analytical data; found: C, 59.89, H, 3.55, F, 5.00; N, 13.05\%. Calculated for $\mathrm{C}_{37} \mathrm{H}_{28} \mathrm{~F}_{2} \mathrm{~N}_{7} \mathrm{PO}_{6}$ (735); C, 60.40; H, 3.80; F, 5.17; N, 13.13\%. IR vcm ${ }^{-1}$ : 3600 - 3200 (b, OH, NH, NH), 1592 (CONH), 1480 (deform. CH=C), 1240 (C-F), 1170 (P=O), 1050 (Ph-O-P), 850, 752 (aryl CH).

3-[5'-(4'’-Fluorophenylidene)-3'-phenyl-1'H-6'-oxo-1',2',4'-triazin-2'-yl]-6-(5'-fluoro-2'-diphenylphosphorylanilido)-1,2,4-triazin-5-(2H)one (21)

Compound 20 (1 mg) and glacial acetic acid (10ml) reflux for 2h, cooled then poured onto ice. The solid thus obtained filtered off and crystallized from ethanol to give 21 as deep yellowish crystals. Yield 80\%, m.p. $233^{\circ} \mathrm{C}$ $235^{\circ} \mathrm{C}$, Analytical data; found: C, 61.81; H, 3.49; F, 5.09; N, 13.55\%. Calculated for $\mathrm{C}_{37} \mathrm{H}_{26} \mathrm{~F}_{2} \mathrm{~N}_{7} \mathrm{PO}_{5}(717)$; $\mathrm{C}_{\text {, }}$ 61.92; H, 3.62; F, 5.29; N, 13.66\%. IR $v \mathrm{~cm}^{-1}$ : $3192(\mathrm{NH}), 2880,2810$ (aliphatic $\mathrm{CH}$ ), 1699 (C=O), 1632 (CONH), 1479 (deform. CH=), 1303 (N-N), 1240 (C-F), 1210 (P=O), 1056 (Ph-O-P), 900, 870, 810, 801 (aryl $\mathrm{CH})$.

6-[5'-fluoro-2'-(diphenylphosphato)aminophenyl]-3,3,3-triphenyl-3- $\lambda^{5}$-1,2,4,3-triazaphopholino[4,5-b] $[1,2,4]$ triazine-7(8H) one $(22)$

A mixture of compound $2(0.01 \mathrm{~mol})$ and triphenyl phosphine $(0.01 \mathrm{~mol})$ in acetonitryl $(20 \mathrm{ml})$ warm for 30 min, cooled. The solid obtained filtered off and crystallized from dioxan to give 22 as deep yellow crystals, yield $70 \%$, m.p. $283^{\circ} \mathrm{C}-285^{\circ} \mathrm{C}$. Analytical data; found: C, 64.01; H, 4.15; F, 2.39, N, 11.35\%. Calculated for $\mathrm{C}_{39} \mathrm{H}_{31} \mathrm{FN}_{6} \mathrm{P}_{2} \mathrm{O}_{4}$ (728); C, 64.28; H, 4.25; F, 2.60; N, 11.53\%. IR vcm ${ }^{-1}$ : 3300 - 3100 (b, NH, NH), 1694 (C=O), $1620(\mathrm{C}=\mathrm{N}), 1307(\mathrm{~N}-\mathrm{N}), 1264$ (C-F), 1179 (P=O), 1027 (Ph-O-P), 910, 880, 793, 745 (aryl CH).

\section{Results and Discussion}

$\alpha$-Aminophosphonic acids continue to elicit study due to interest in their biological properties as herbicides [22], plant growth regulators [23] and most notably those species heaving a direct P-N bond are investigated as transition state analogues of the tetrahedral transition-state involved in peptide hydropysis [24]. Ali et al. [25] [26] studied the reactivity of $\alpha$-amino phosphonates as dipolar ion structure and have type of tautomeric formula due to the higher e-withdrawing of two phenoxy and $\mathrm{P}=\mathrm{O}$ groups (Figure 2). Thus, $\alpha$-aminophosphonate group had 


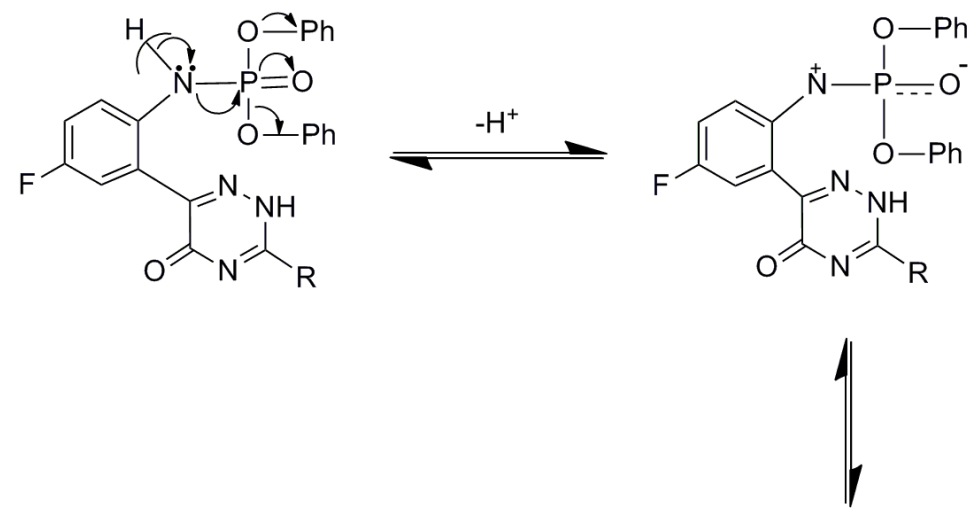<smiles>[R]c1nc(=O)c(-c2cc(F)ccc2N=P(Oc2ccccc2)(Oc2ccccc2)Oc2ccccc2)n[nH]1</smiles>

Figure 2. A possible present formula of the new synthesized isolated systems.

a higher degree of stability towards any attack of reagents, which attribute to presence of differ factors of stability [27]. Phosphorus elements in these systems, was determine by using the spectrophotomeric. The method is based on the development of floated complex of molybdophosphonic acid (MPA) and methylene blue (MB) with $\mathrm{N}, \mathrm{N}^{\prime}$-diphenylbenzamide (DPBA) in toluene and its subsequent dissolution in acetone [28].

A series of some new fluorine substituted phosphoryl-amino-1,2,4-trinzines bearing a functionally pyrazole ring have been obtained via cycloaddition and/or cyclocodensation of 3-hydrazino-1,2,4-trinzinone3 with $\pi$-acceptor activated carbon atom reagents. In addition, a type of 1,2,4-trinzino [4,3-b] [1,2,4] triazindiones have been also obtained from cyclocondensation of compound 3 with $\alpha, \beta$-bifunctional oxygen and halogen compounds in different conditions. The former structures of the new products confirmed from correct elemental analysis and their spectral measurements. Keeping in view the diverse medicinal activities associated with organo-heterocyclilc systems substituted fluorine, phosphorus and 3-thioxo-1,2,4-trinzinone, which intend to construct novel fluorinated substituted phosphoryl amino bearing 1,2,4-triazinone moiety hoping to active additive effects towards their HIV-1 activity.

\subsection{Chemistry}

The starting material 3-hydrazino-6-(5'-fluoro-2'-diphenylphosphorylanilido)-1,2,4-trizino-5(2H) one (3) obtained from treatment of 6-(5'-fluoro-2'-aminophenyl)-3-thioxo-1,2,4-triazin-5(2H)one (1) with diphenylphosphoryl chloride in DMF to give 6-(5'-fluoro-2'-diphenylphosphorylanilido)-3-thioxo-1,2,4-triazin-5-one (2) followed by hydrozinolysis in boiling ethanol (Scheme 1).

Recently, the most reactions of activated nitrites take place in basic medium leading to novel heterocyclic systems [29] [30]. Similarly, amino-pyrazolyl-1,2,4-triazinano derivatives 4-7 were obtained from the interaction between compound 3 and arylidenecyanoacetic acid (EtOH-piperidine), acetyl acetanilide (DMF), ethyl cyanoacetate (TFH) and/or malono nitrile (EtOH-piperidine) (Scheme 2).

These reactions are carried out via cycloaddition and/or cyclocondensation reactions [31] (Figure 3).

It is interested that 3-perhydropyrazo-1-yl-1,2,4-trizinones 8-10 also obtained from the ring closure reaction of compound 3 with cinnmoyl chloride (DMF), chalcone (EtOH-piperidine) and or diethylmalonate (THF) (Scheme 3).

Formation of 8 may be take place via aroylation then cycloadditon reaction [32] (Figure 4).

The greater reactivity of the polyfunctional compound as aroylisothiocynate towards the hydrazino group as bi-nucleophile is presumably due to its favourable location between both carbonyl and thiocarbonyl functional 


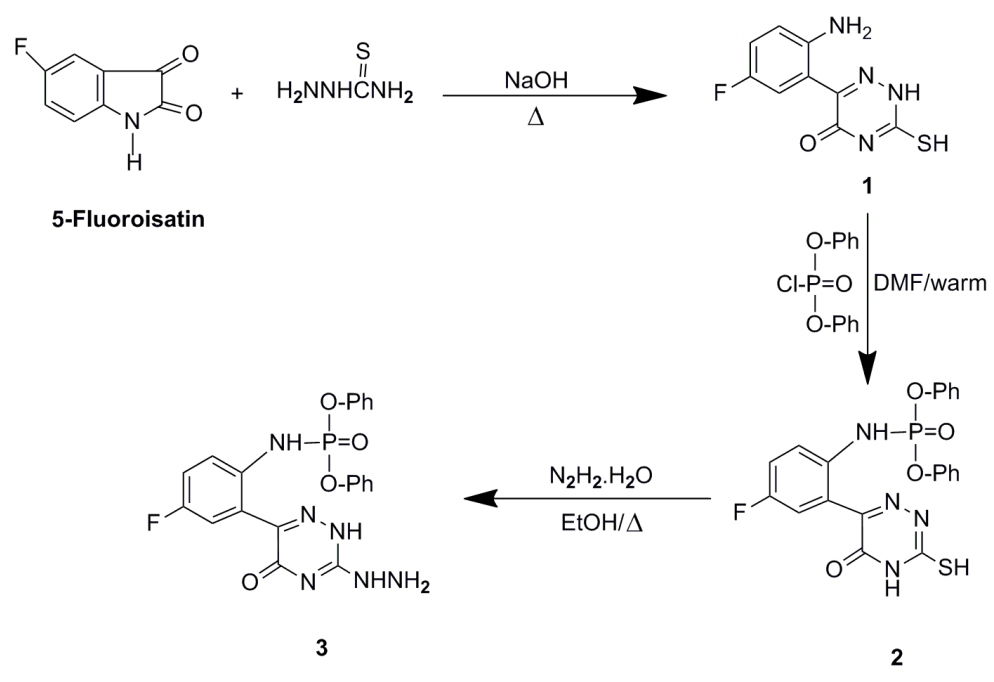

Scheme 1. Formation compounds 1-3.

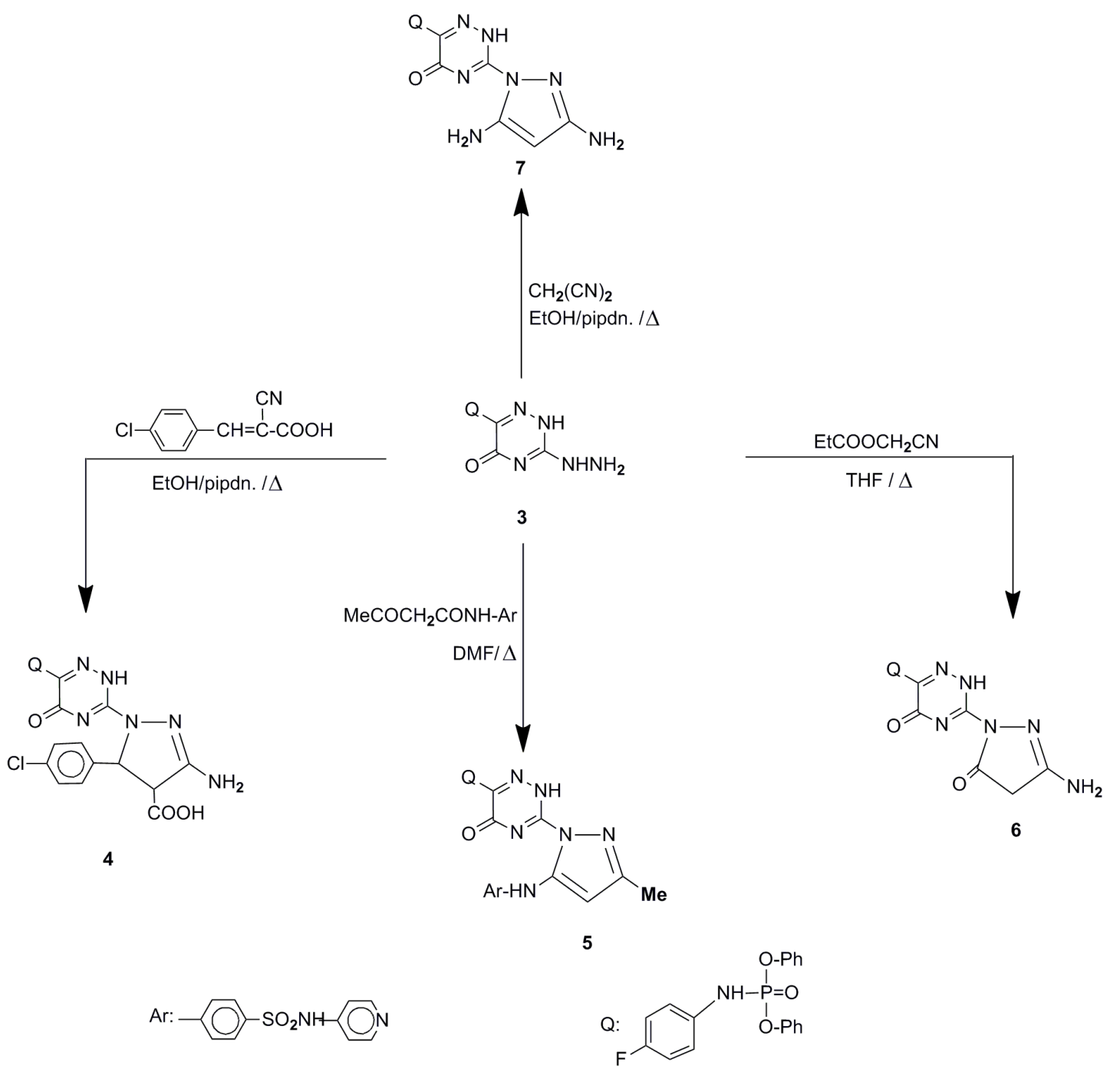

Scheme 2. Formation compounds 4-6. 

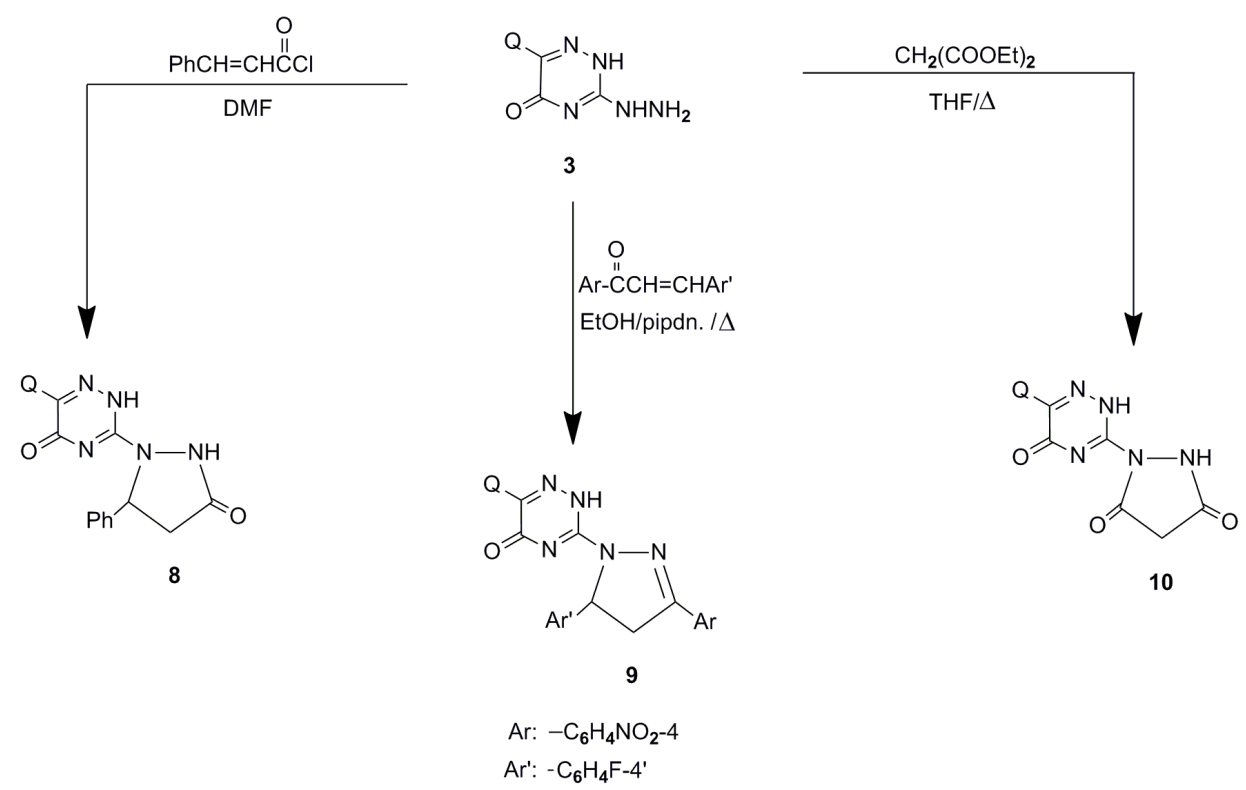

Scheme 3. Formation compounds 8-10.

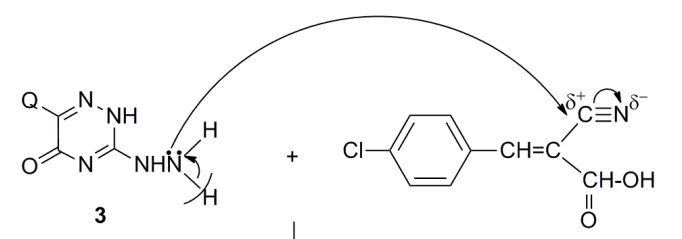

A

B

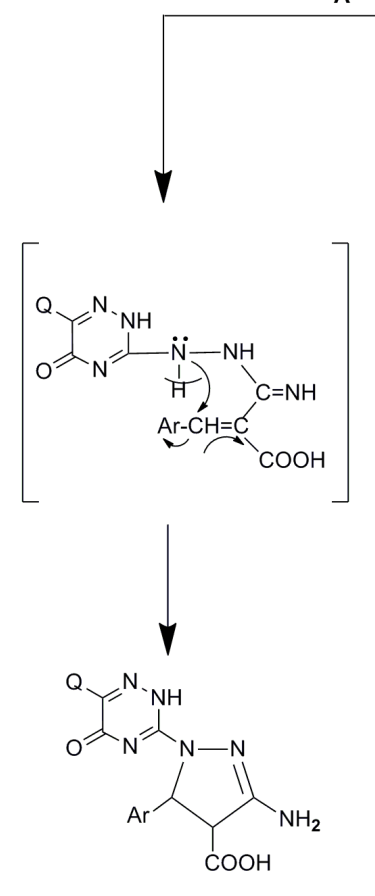

4 (A)
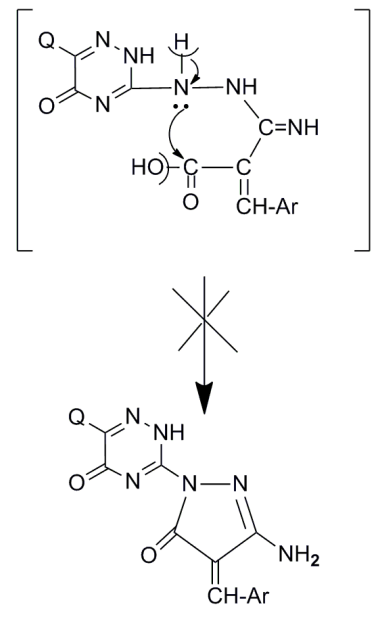

4 (B)

Figure 3. Formation of compound 4 from compound 3. 


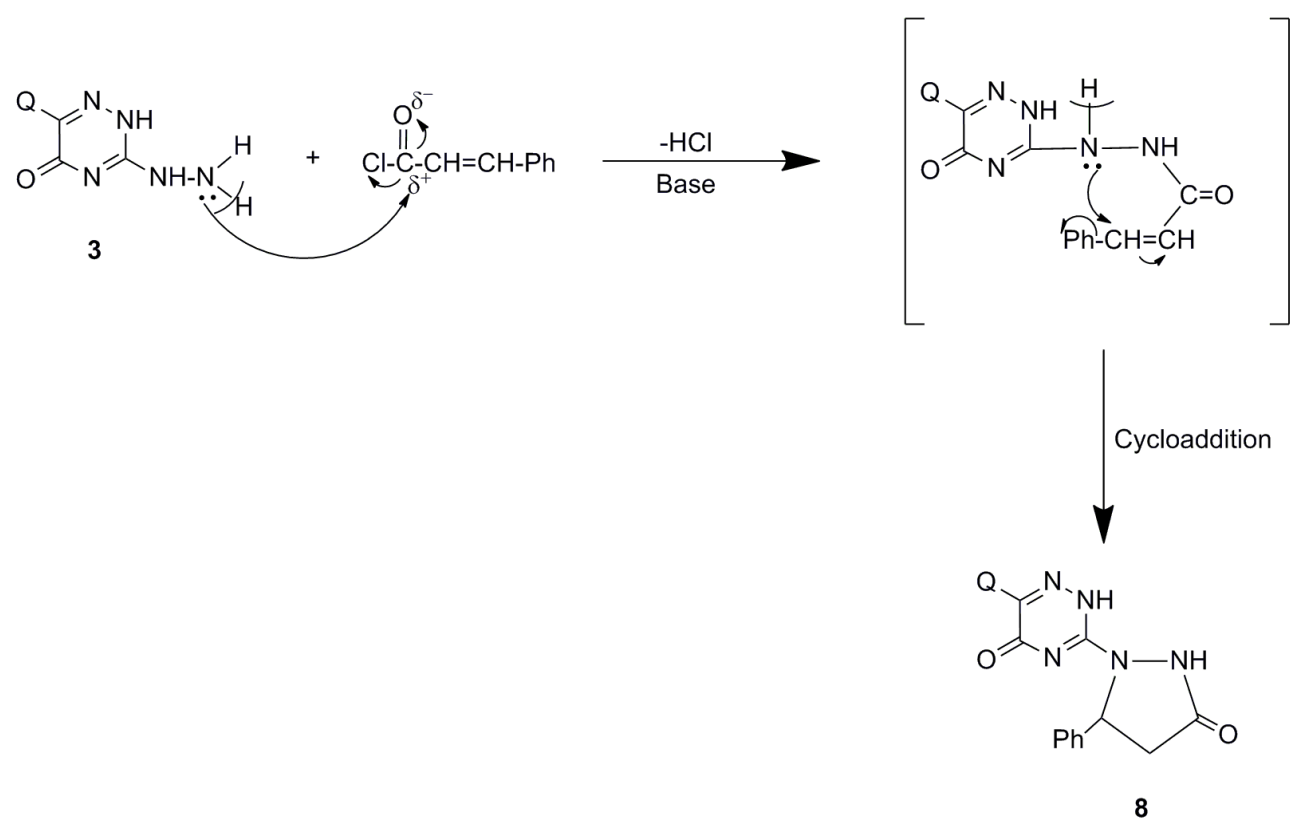

Figure 4. Formation of compound 8 from compound 3.

groups [33]. Thus, treatment of 3-hydrazino-1,2,4-trizinone 3 with aroylisothiocyanate in boiling non-polar solvent as dioxan yield 3-(5'-aryl-3'-mercapto-1',2',4'-triazol-1'-yl)-6-(5'-fluoro-2'-diphenly phosphorylanilido)-1, 2,4-triazin-5(2H)one (11), while that reaction when carried out in DMF, 3-(3'-aryl-5'-mercapto-1', 2',4'-triazol-1'-yl)-6-(5'-fluoro-2'-di-phenylphosphorylanilido)-1,2,4-trizin-5-(2H) one (12) isolated (Scheme 4). Formation of compounds $\mathbf{1 1}$ and $\mathbf{1 2}$ starting from compound $\mathbf{3}$ were outlined in (Scheme 4).

Reactivity of $\alpha, \beta$-bifunctional carbonyl compounds towards an hydrazino groups arrived us to synthesize new fused heterobicyclic nitrogen systems. Thus, the interaction between compound $\mathbf{3}$ and sodium pyruvate in warming sodium hydroxide solution afforded 1H-3-methyl-7-aryl-1,2,4-triazino[4,3-b][1,2,4] traizin-4,8-dione (13), while cyclo-condensation of compound 3 with isatinas 1,2-bicarbonyl compound in boiling DMF yield indolo [2,3-e] [1,2,4] trinzino [4,3-b][1,2,4] triazin one (14). Refluxing of 3 with phenacyl bromide in ethanolic $\mathrm{KOH}$ furnish the tetrahydro-1,2,4-triazino [4,3-b][1,2,4] triazin-8-one (15) (Scheme 5).

A large degree of the biological activity is attributed of the nature of substituent's and a degree of electronic distribution over the active center of the 1,2,4-triazines [34] [35]. Thus, direct nucleophilic displacement of chlorine atoms by nitrogen or other nucleophilic can easily occur if present $\alpha$-carbonyl groups. Based on these facts, treatment of compound 3 with activated halogen as chloroacetic acid (DMF), dichloroacetic acid (DMF) and or oxalyl chloride $\left(\mathrm{C}_{6} \mathrm{H}_{6} / \mathrm{TEA}\right)$, produce perhydro 1,2,3,4-tetrahydro-1,2,4-triazino [4,3-b] [1,2,4] triazin4,8-dione (16); 1,2-dihydro-1,2,4-triazino [4,3-b] [1,2,4] triazin-4,8-dione (17) and 1,2,3,4-tetrahydro-1,2, 4triazino [4,3-b] [1,2,4] triazin-3,4,8-trione (18) derivatives (Scheme 6).

In view of interesting results obtained from the reaction of 1,3-oxazolium salts and of 1,3-oxazol-2-one derivatives with hydrazine derivatives[36], [37], it was worthwhile to investigate the behavior of oxazolone19 towards hydrazine-derivative. Similarly, 3-hydrazino-6-aryl-1,2,4-triazin-5 (2H) one (3) when react with oxazole derivation 19 in boiling aqueous ethanol, the acid hydrazide derivative 20 isolated. Ring closure reaction of 20 by refluxing with glacial acetic acid afforded 3-(1'H-3-phenyl-5'-arylidene-6'-oxo-1,2,4-triazin-2'-yl-1-6-(5'-fluoro2'-diphenylphosphorylanilido)-1,2,4-triazin-5(2H)one(21)Finally,6-[5'-fluoro-2'-(diphenylphosphato)aminoph-enyl]3,3,3-triphenyl-3- $\lambda^{5}-1,2,4,3$-triazaphopholino[4,5-b][1,2,4]triazine-7(8H) one (22) isolated from treat compound 3 with triphenylphosphine in THF (Scheme 7).

\subsection{Elucidation the Former Structures}

\subsubsection{UV Spectra Study}

UV absorption study of the new compounds, synthesize give us a good indication about electronic distribution 


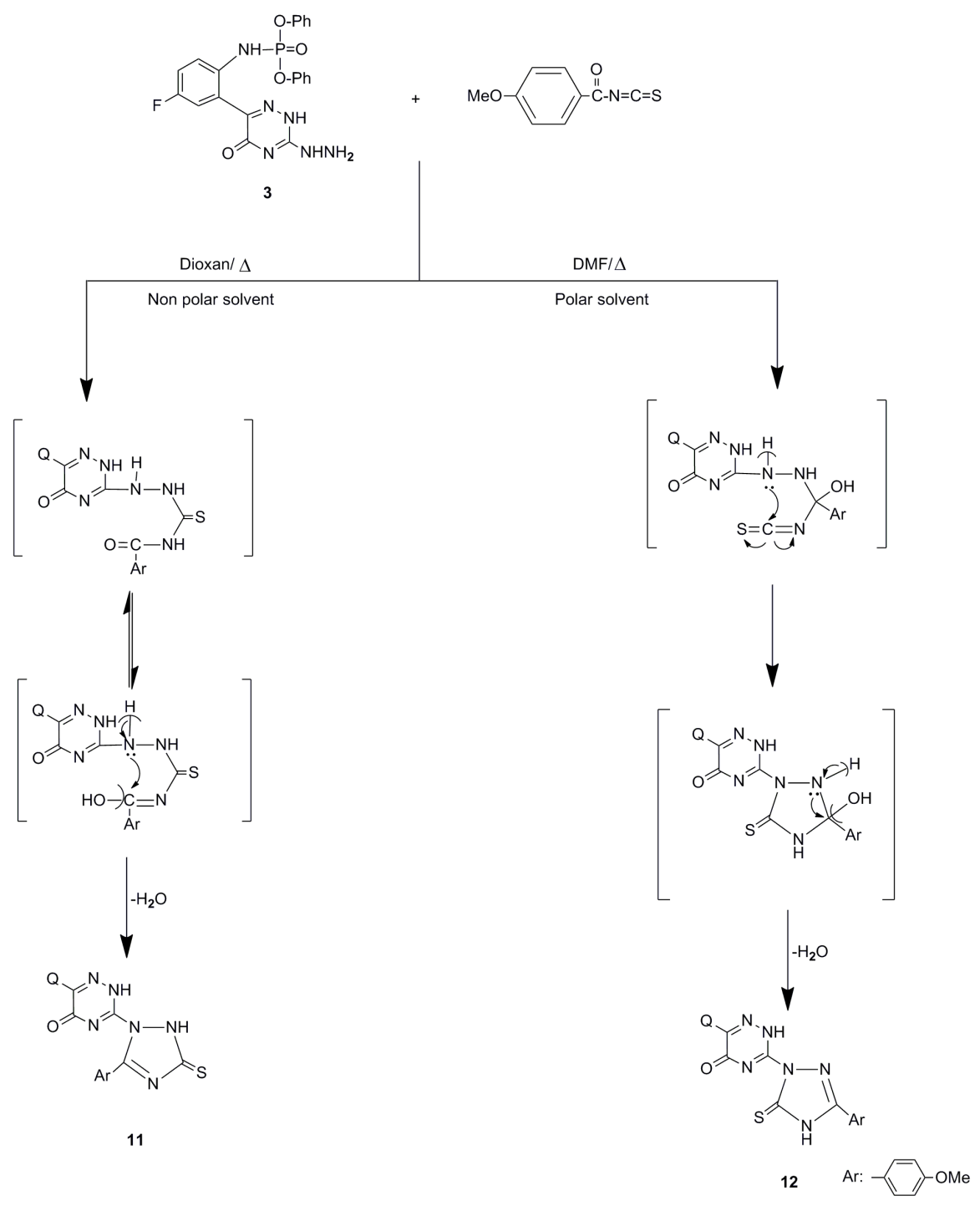

Scheme 4. Formation compounds 11 \& 12.
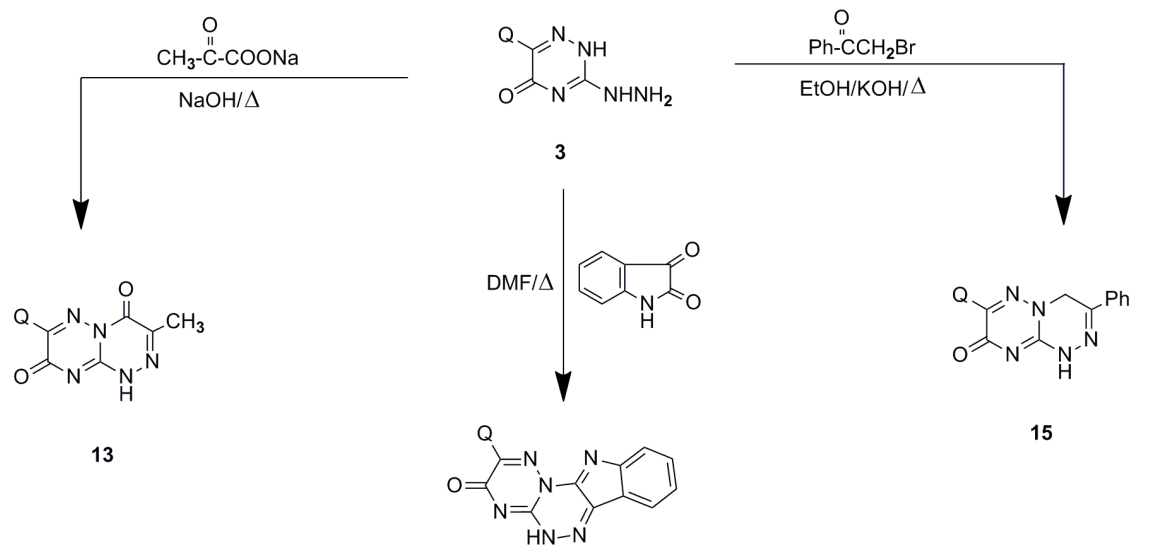

14

Scheme 5. Formation compounds 13-15. 


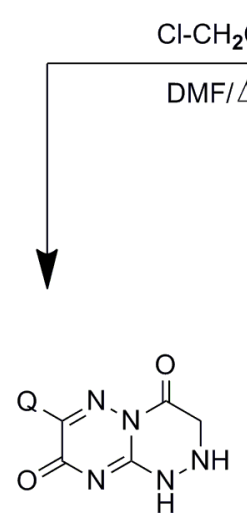

16

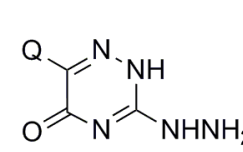

3

$\mathrm{Cl}_{2} \mathrm{CHCOOH}$

DMF/ $\triangle$

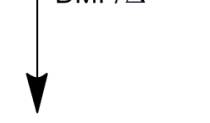<smiles></smiles>

$\mathrm{COCl}$

$\mathrm{COCl}$

$\mathrm{C}_{6} \mathrm{H}_{6} /$ TEA

17

Scheme 6. Formation compounds 16-18.<smiles>NNc1nc(=O)c(O)n[nH]1</smiles>

3<smiles>O=C1CCCCC1=Cc1ccc(F)cc1</smiles>

19

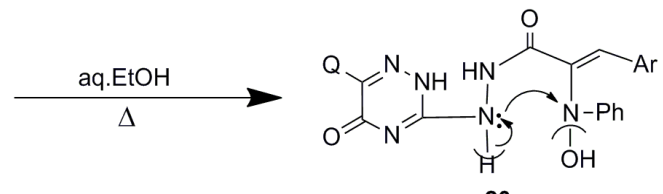

20

$\mathrm{AcOH}$

$-\mathrm{H}_{2} \mathrm{O}$<smiles>O=C1NN(c2nc(=O)c(O)n[nH]2)C(c2ccccc2)=N/C1=C\c1ccc(F)cc1</smiles>

21<smiles>NNc1nc(=O)c(O)n[nH]1</smiles>

3

22

Scheme 7. Formation compounds 19-22.

and molecular configuration as possible.In general, all the new compounds record the presence of n- $\pi^{*}$, n- $\sigma^{*}$, $\pi-\pi^{*}$ and $\sigma-\sigma^{*}$ electronic transition. UV-absorption spectrum of compound 3 as state recorded $\lambda_{\max }(\mathrm{EtOH})$ at $346.48 \mathrm{~nm}$, while that of compounds 14 (361.55) and 12 (361.12). A higher value of $\lambda_{\max }$ for 14 and 12 than 3 is may be a lack's of -OH groups (which generate of H-bending (Figure 5).

On the other hand, UV absorption spectra of selected compound record a lower $\lambda_{\max }$ than compound 3. $\lambda_{\max }$ of 10 (330.56) and 18 (317.22) nm. A lower $\lambda_{\max }$ of these compounds than the start is may be due to the presence of $\mathrm{O}-\mathrm{H}$ group, which generate a type of H-bonding. The Keto-enol forms of $\mathbf{1 0}$ and $\mathbf{1 8}$ led to the inhibition of heteroconjugative, in addition a type of H-bonding which is closed to 1,2,4-triazine moiety (Figure 6). 


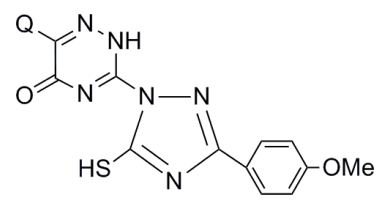

$12 \lambda_{\text {max }}=361.12 \mathrm{~nm}$

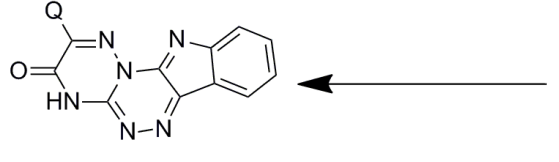

$14 \lambda_{\max }=361.55 \mathrm{~nm}$<smiles>NNc1nc(=O)c(-c2cc(F)ccc2NP(=O)(Oc2ccccc2)Oc2ccccc2)n[nH]1</smiles>

$3 \lambda_{\text {max }}=346.48 \mathrm{~nm}$

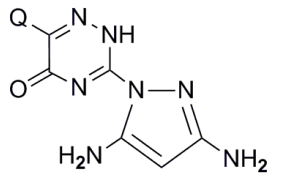

$7 \lambda_{\max }=346.05 \mathrm{~nm}$

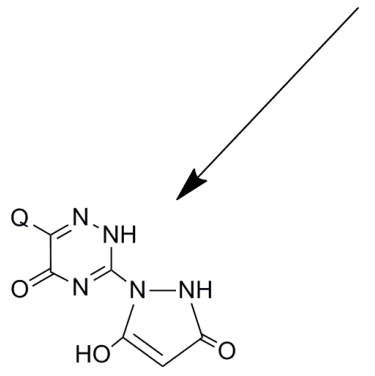

$10 \lambda_{\text {max }}=330.56 \mathrm{~nm}$

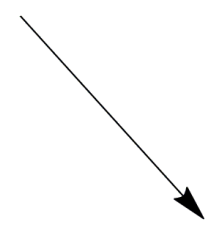

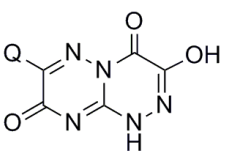

$18 \lambda_{\max }=317.22 \mathrm{~nm}$

Figure 5. UV-absorbtion data of compound 3 and some prepared compounds.<smiles>O=c1nc2[nH][nH]c(=O)c(=O)n2nc1-c1cc(F)ccc1NP(=O)(O)Oc1ccccc1</smiles>

18<smiles>O=c1nc2[nH]nc(O)c(=O)n2nc1-c1cc(F)ccc1NP(=O)(Oc1ccccc1)Oc1ccccc1</smiles>

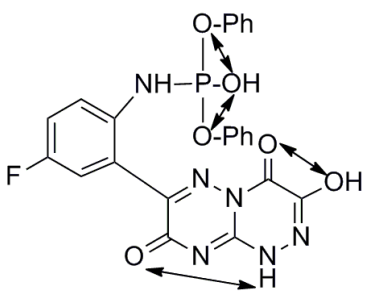

Figure 6. A possible conformer structure of 18 (fused system).

\subsubsection{IR-Absorption Study}

The IR absorption spectral data show that most of the new compounds lack's a band of NH-P=O, which is due to a type of H-bonding present (Figure 6), while that of these compounds record only NH bond of new 1,2, 4-triazinones and/or pyrazoles moiety. In addition, IR absorption spectra of all the synthesized compounds exhibit an absorption bands at 3300 - $3190(\mathrm{NH}), 1690$ - $1650(\mathrm{C}=\mathrm{O})$. Moreover presence of a characteristic bands at $v 1390-1370$ for cyclic NCN, 1250 and $1220-1200 \mathrm{~cm}^{-1}$ for C-F and $\mathrm{P}=\mathrm{O}$ functional. Also, all the new compounds record $v$ at $1100-1050 \mathrm{~cm}^{-1}$ attribute to Ph-O-P group. Only, the compounds 3, 4, 6, and 7 record a $v$ for 
$\mathrm{NH}_{2}$ at $3390-3400 \mathrm{~cm}^{-1}$, while that of the compounds $4, \mathbf{6}, \mathbf{8}, \mathbf{1 0}, \mathbf{1 3}, \mathbf{1 6 - 2 1}$ showed $v$ for $\mathrm{C}=\mathrm{O}$ in addition the original $\mathrm{C}=\mathrm{O}$ of 1,2,4-trinzine. Finally, IR spectra of the compounds 4, 5, 6, 8-13 and 15, 16 record a type of bands characteristic for aliphatic groups (deformation $1480-1440 \mathrm{~cm}^{-1}$ ).

\subsubsection{NMR Spectral Study}

\section{1) ${ }^{1}$ H NMR Spectral Study}

The NH proton signals in all the new compounds appear as doublets at $\delta 8.8-8.2 \mathrm{ppm}\left(\mathrm{JP}-\mathrm{N}-\mathrm{H}=6-5.5 \mathrm{H}_{\mathrm{z}}\right.$ ) is due to its coupling with phosphorus. Also, $\mathrm{H}$-bonding with oxygen of $\mathrm{P}=\mathrm{O}$ and the deshielding effect of phenoxyphosphoryl group (Ph-O-P=O) are obviously the contributing factors for the downfield shift of the $\mathrm{NH}$ proton. On the other hand, phenoxy protons resonated at $\delta 7.50-7.1 \mathrm{ppm}$ and their integration corresponds to five protons with no splitting of the signals. This shows that all the protons as magnetically equivalent. Normally, exo and endo $\mathrm{NH}$ protons of 1,2,4-triazinone reveal at $\delta 12$ - $11 \mathrm{ppm}$, while what of the 1,2,4-tiazinone addujent of $\mathrm{CH}_{2}$ or $\mathrm{NH}$ protons show as enolic protons at $\delta 11-10 \mathrm{ppm}(\mathbf{5 , 7 , 1 7 , 1 8 , 9 ) . I n ~ a d d i t i o n , ~ a l l ~ t h e ~ n e w ~ c o m p o u n d s ~}$ 4 \& 6, exhibited a resonated signals at $\delta 5-4 \mathrm{ppm}$ as $\mathrm{NH}_{2}$ protons and $\mathrm{NH}$ proton at $\delta 11-10 \mathrm{ppm}$. Finally, the perhydro pyrazolyl-1,2,4-triazinones and the 1,2,4-trinzino-1,2,4-trinzinones which containing an aliphatic protons show a resonated signals at $\delta 4-3$ and $1-0.5 \mathrm{ppm}$ for $\mathrm{CH}_{2}$ and $\mathrm{CH}_{3}$ protons.

\section{2) ${ }^{13}$ CNMR Spectral Study}

The ${ }^{13} \mathrm{C}$ Chemical shifts of phenoxy moiety are agreeing well with the reported values [38]. But, the coupling constants for ${ }^{2}$ Jare concurring with those of equationally oriented P-O-Ar groups [39] showing the 1,2,4-trinzine ring has probably half chair conformation with phosphorus atom projecting upwards and the O-Ar group orienting equationally. The carbons $\mathrm{C}$, which are connected to phosphorous through $\mathrm{NH}$, resonated at $\delta 112.97$ with ${ }^{2} \mathrm{~J}$ $\mathrm{P}-\mathrm{N}-\mathrm{C}(\mathrm{d}, \mathrm{J}$ 8.1) and the difference in their chemical shifts may be attributed to the variation of shielding effect of $\mathrm{NH}$ [40]. In addition, all the new compounds record the resonated attribute to C=O (170 - 160), C-F (150 $140), \mathrm{C}=\mathrm{N}(140-130)$, $\mathrm{C}-\mathrm{N}(111-110)$ of 1,2,4-triazinone, with a differ type of carbons of pyrazolyl as well as carbons of other 1,2,4-triazine formed (Figure 7 and Figure 8).

\subsubsection{Mass Spectrometric Measurements}

The mass spectral investigations of the isolated heterobicyclic system is differ than the fused heterobicyclic systems (14 \& 18) for example, $M / Z$ of 7 and/or 10 recorded the molecular ion Peak's at m/z 534 and 336 respectively with a lower abounds percent's, which indicating the fragile nature of these systems. While M/Z of compound $\mathbf{1 4}$ showed the highest value peak at 579 with a base peak at $\mathrm{m} / \mathrm{z} 95$, which give us a high degree of stability for this Skelton. Moreover, M/Z of 18 exhibits a molecular ion peak at m/z 522 with moderate abounds percent. From these data, we can conclude that fused heterobicyclic nitrogen systems are more stabilized [41]

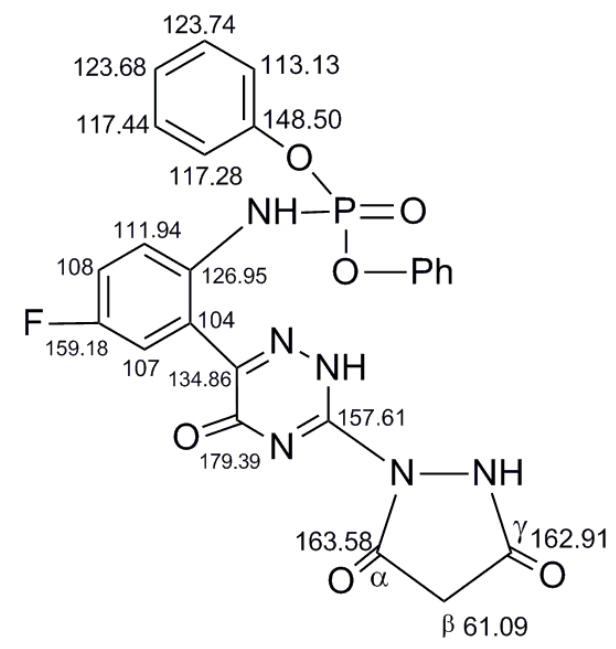

$\delta$ in ppm and $\mathrm{J}$ in $\mathrm{H}_{\mathbf{Z}}$ 
than other isolated heterobicyclic systems. It's interest that in all mass fragmentation pattern, 4-fluorophenyl ion is a base peak followed by N-phosphorus oxide ions, while that heterocycle supported that a large fragmentation bath way (Figures 9-12).<smiles>COc1ccccc1</smiles><smiles>Cc1nc(=O)c(-c2cc(F)ccc2NP(C)(=O)Oc2ccccc2)n[nH]1</smiles><smiles>CN1NC(=O)CC1=O</smiles>

$\delta$ in ppm and $\mathrm{J}$ in $\mathrm{H}_{\mathbf{Z}}$

10

Figure $8 .{ }^{13} \mathrm{C}$ NMR data of compound 18.

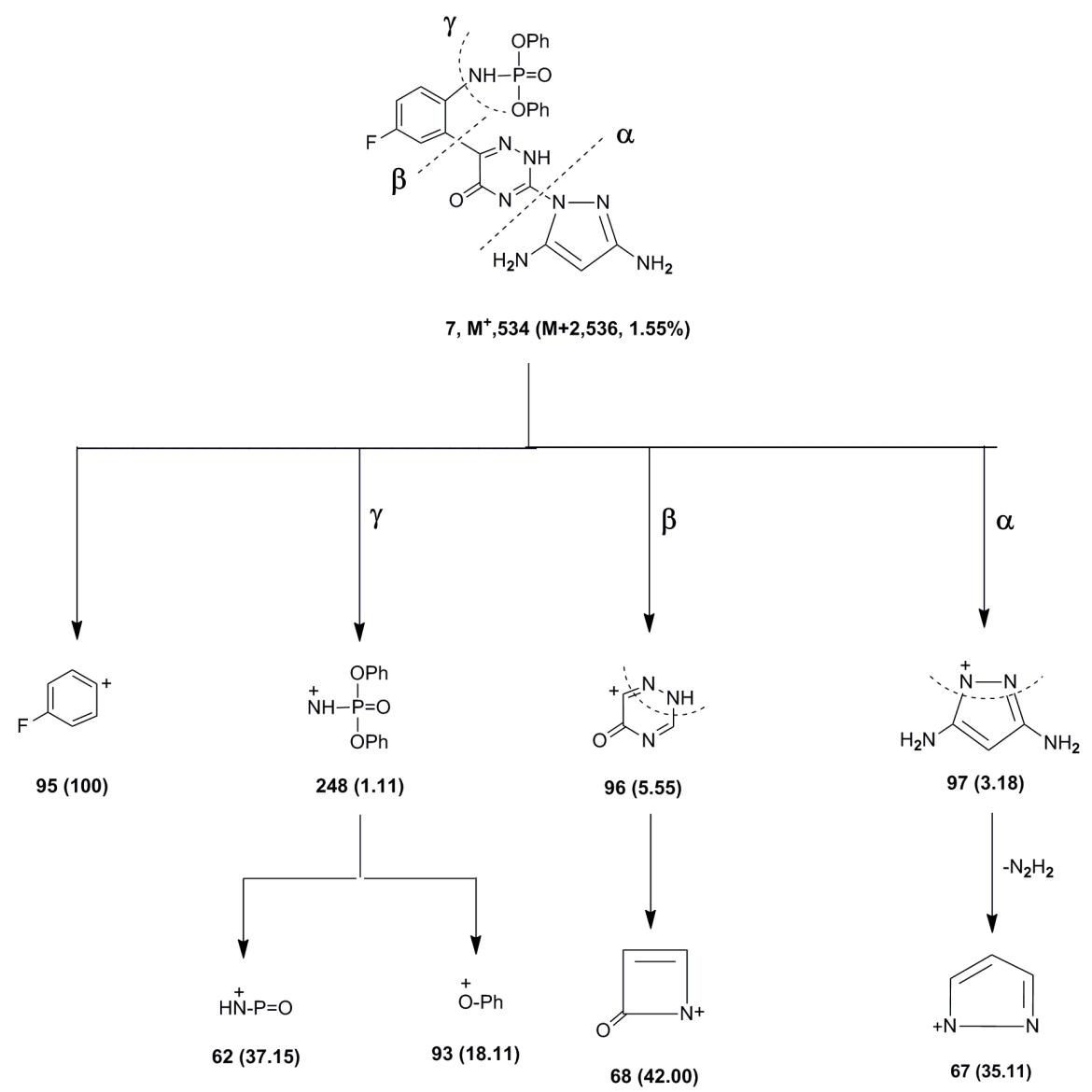

Figure 9. Mass Fragmentation pattern of compound 7. 


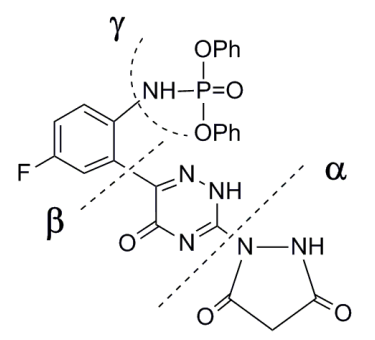

$10, \mathrm{M}^{+}, 536(2.28 \%)$

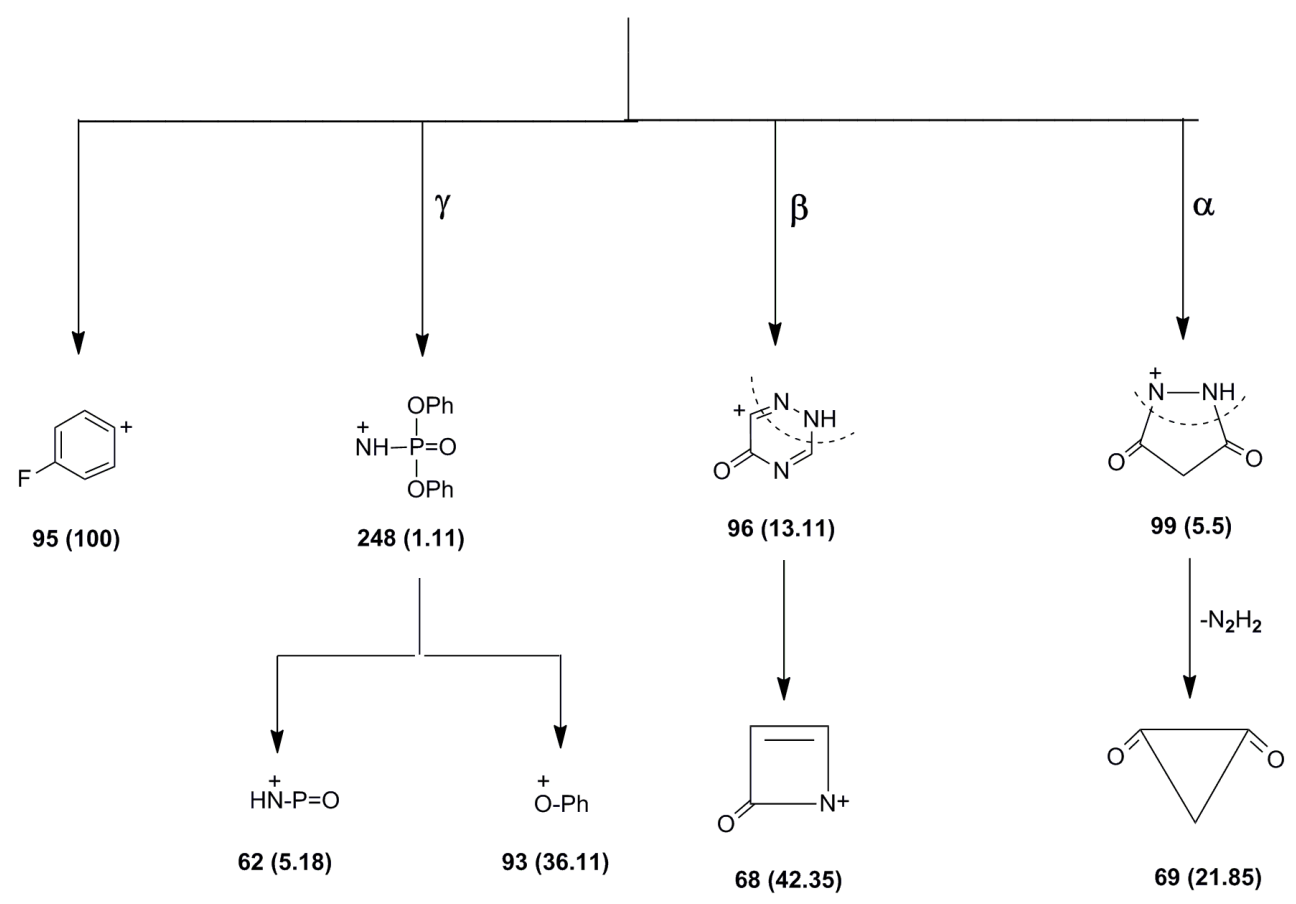

Figure 10. Mass Fragmentation pattern of compound 10.

\section{HIV-1 Inhibition (Enzyme Inhibition)}

Human immunodeficiency virus type- 1 is the causative agent of acquired immunodeficiency syndrome (AIDS) which is one of the most serious health problems [42]. Since reverse transcriptase (RT) is an essential enzyme for the replication [43] of HIV, it is the most favoured target for the antiviral chemotherapy against HIV infection [44]. 3'-Azido-2',3'-dideoxythymidine (AZT) [45] and 2',3'-dideoxyinosine (DDI) [46], 2',3'-dideoxycytydine (DDC) [47] and 2',3'-didehydro-3-deoxythymidine (DT4) [48] are the well-known potent nucleoside reverse transcriptase inhibitors clinical use, but unfortunately they produce serious side effects such as bone marrow suppression. The search for a more effective and less toxic agent has brought into focus potent yet structurally different non-nucleoside HIV-1 reverse transcriptase inhibitors (NNRTIs) [49]. Shakil et al., [50] reported that increase or decrease of electro-negativity and hydrophobicity of the bioactive drugs, cytotoxicity will also increase or decrease accordingly. So less electronegative and less hydrophobic substituents would be preferred to design the less cytotoxic drugs. The large number of research papers published every year indicate that the development of an effective drug for the inhibition of HIV-1 via enzymes inhibitors [51] [52]. HIV PIs for example, prevent the cleavage of the gag and gag-pol precursor polyproteins to the structural proteins and functional proteins, thus arresting maturation and thereby blocking infectivity of the nascent virions. e.g. Tipranavir showed loss cross-resistance to HIV strains that were resistant to the established (peptidomimetic) inhibitors of HIV protease. Also, tipranavir retained marked activity against HIV-1 isolates derived from patients with multidrug resistance to other PIs (Figure 13). 


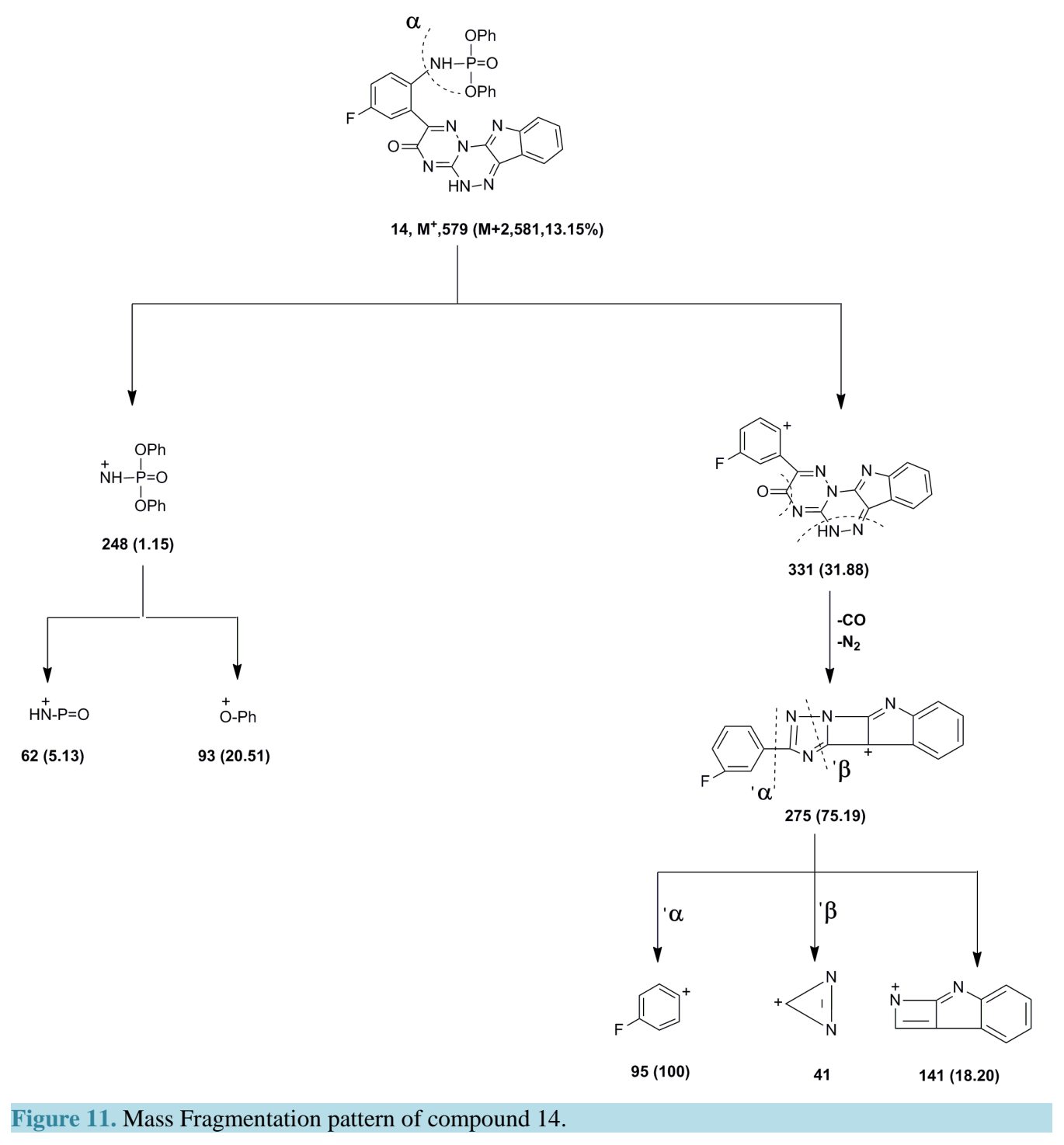

In search for new poly substituted 1,2,4-triazine bearing a phosphoryl group. The present work is synthesize novel fluorine substituted phosphorylanilido-1,2,4-triazin-ones and evaluate as potential inhibitors for HIV-1. The procedure used in the National Cancer Institute's Test for agents active against HIV is designed to detect agents acting at any stage of the virus reproductive cycle [53]. The assay basically involves the killing of $\mathrm{T}_{4}$ lymphocytes by HIV. Small amounts of HIV are added to cells and two cycles of virus reproduction are necessary to obtain the required cell killing. Agents that interact with virions, cells or virus gene-products to interfere with viral activities will protect cells from cytolysis. The tetrazolium salt XTT is added to all wells and cultures are incubated to allow formazan color development by viable cells used analyzed spectrophotometrically (Figure 14).

Anti-HIV-1 screening results of some of compounds (Table 1) shows that these systems found inactive, probably due to their inability to exist in butterfly like conformation as explained in a similar case [54]. Only the compounds 7, 12, 18 and 22 exhibited a higher protection\% (concentration required in protect MT-4 cells against the cytopathogenicity of HIV by 50\%). The order reactivity increases as $18>10>7>12>22$. A higher effect's of compound 18 (Table 2) is may be due to a higher possibility to form a type of H-bonds with proteins of virus, which led to a moderate degree of inhibition of HIV-1 activity. Also, compound $\mathbf{1 8}$ had a differ type of phenolic bonds, which give a variety of differ effects towards HIV-1 activity. Moreover, compound $\mathbf{1 8}$ had a less possibility to form a type of intra-molecular H-bond, thus their hydroxyl group become a higher degree of free action 


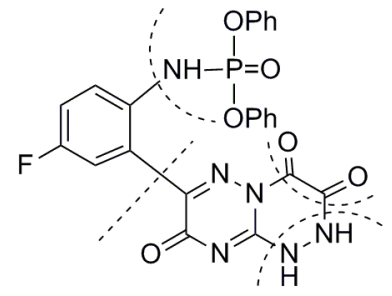

$18, M^{+}, 522(M+3,525,18.55 \%)$

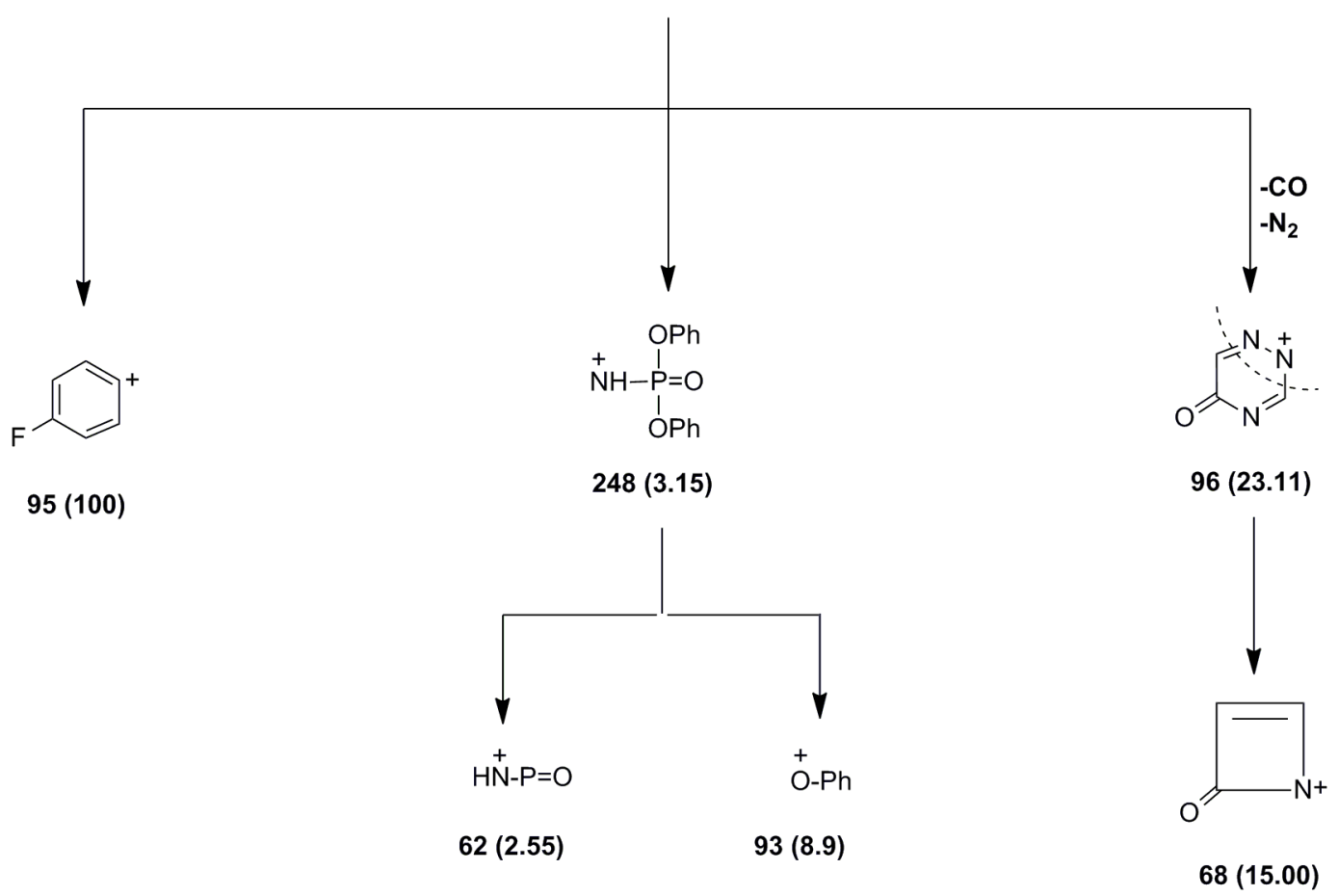

Figure 12. Mass Fragmentation pattern of compound 18.

Table 1. The in vitro anti-HIV-1 screening results of new compounds 3-22 in MT-4 cells.

\begin{tabular}{|c|c|c|c|c|c|}
\hline \multirow{3}{*}{ Compd. No. } & \multirow{3}{*}{$\begin{array}{l}\text { Dose (Molar) } \\
\text { 2. } 00 \times 10^{-6}\end{array}$} & \multicolumn{4}{|c|}{ Anti-HIV-1 Activity } \\
\hline & & \multirow[t]{2}{*}{$\mathrm{IC}_{50}(\mu \mathrm{g} / \mathrm{mL})$} & \multirow[t]{2}{*}{ Max Production \% } & \multicolumn{2}{|c|}{ Percent of Control } \\
\hline & & & & Infected & Uninfected \\
\hline & 3 & $>55.13$ & 13.5 & 10.11 & 101.11 \\
\hline & 6 & $>20.20$ & 19 & 17.38 & 106.32 \\
\hline & 7 & $>27.20$ & 26.5 & 63.51 & 97.93 \\
\hline & 10 & $>19.40$ & 30.5 & 80.29 & 96.11 \\
\hline & 12 & $>28.57$ & 25.0 & 35.40 & 105.77 \\
\hline & 14 & $>88.30$ & 9.5 & 0.04 & 0.51 \\
\hline & 16 & $>3.99$ & 16.00 & 14.16 & 101.91 \\
\hline & 18 & $>42.60$ & 35.00 & 83.47 & 104.97 \\
\hline & 22 & $>94.40$ & 23.5 & 18.19 & 104.12 \\
\hline
\end{tabular}


Table 2. The in vitro anti-HIV-1 screening results of compound 18.

\begin{tabular}{cccccc}
\hline Index & Concentration & Dose & Percent of Protection & \multicolumn{2}{c}{ Percent of Control } \\
\cline { 4 - 5 } & & & & Infected & Uninfected \\
\hline IC $_{50}$ (Molar) & $1.12 \times 10^{-5}$ & $6.33 \times 10^{-9}$ & 7.55 & 13.99 & 101.90 \\
EC $_{50}$ (Molar) & $4.26 \times 10^{-7}$ & $2.0 \times 10^{-8}$ & 10.10 & 17.11 & 106.20 \\
TIC $_{50}$ & $2.63 \times 10^{+1}$ & $6.35 \times 10^{-8}$ & 12.00 & 18.15 & 104.21 \\
& & $2.00 \times 10^{-7}$ & 30.01 & 32.31 & 105.30 \\
& & $6.33 \times 10^{-7}$ & 60.66 & 62.15 & 98.10 \\
& & $2.00 \times 10^{-6}$ & 82.23 & 83.47 & 104.99 \\
& & $6.34 \times 10^{-6}$ & 75.66 & 80.13 & 99.10 \\
\end{tabular}

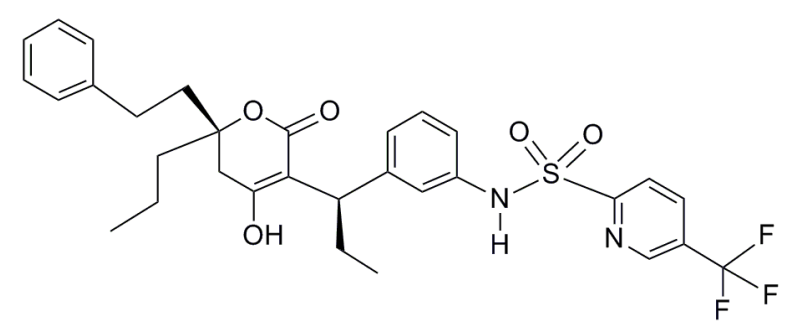

Figure 13. Tipranavir (PNU-140690).
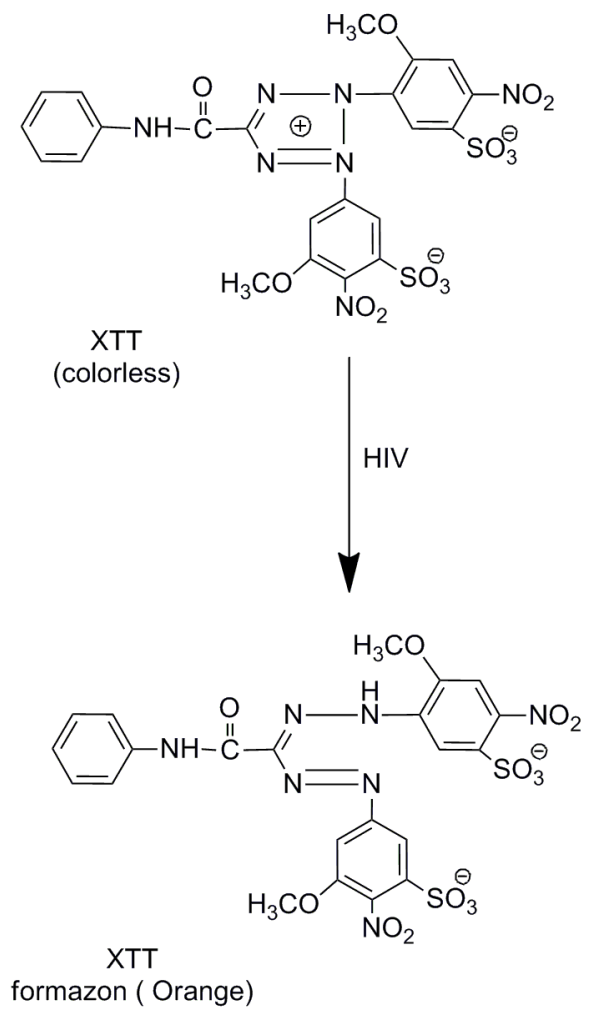

Figure 14. The standard indicator for HIV present (XXT). 
with a active center of proteins of HIV-1 virus.

\section{Conclusion}

In search for new anti-HIV-1, novel fluorine substituted isolated and fused heterobicyclic nitrogen systems bearing 6-(2'-phosphorylanilido)-1,2,4-triazin moiety have been obtained from a ring closure reactions of the corresponding 3-hydrazino-1,2,4-triazinone with $\pi$-acceptable reagents. Some compounds exhibited a mark's inhibitors as anti-HIV-1 activity in hope to a possible control on the HIV-1 activity.

\section{References}

[1] Hartley, F.R. and Dahl, O. (1996) The Preparation and Properties of Tervalent Phosphorus Acid Derivatives. In: Brecuer, E. and Heartly, F.R., Eds., The Chemistry of Organophosphorus Compounds: Ter- and Quinque-Valent Phosphorus Acids and Their Derivatives, John Wiley \& Sons, New York, 653.

[2] Prakasha, T.K., Day, R.O. and Holmes, R.R. (1994) Pentacoordinated Molecules. 101. New Class of Bicyclic Oxyphosphoranes with an Oxaphosphorinane Ring: Molecular Structures and Activation Energies for Ligand Exchange. Journal of the American Chemical Society, 116, 8095-8104. http://dx.doi.org/10.1021/ja00097a016

[3] Fest, C. and Schmidt, K.J. (1982) The Chemistry of Organophosphorus Pesticides. Springer-Verlag, Berlin, 12. http://dx.doi.org/10.1007/978-3-642-68441-8

[4] Bhatia, M.S. and Jit, P. (1976) Phosphorus-Containing Heterocycles as Fungicides: Synthesis of 2,2'-Diphenylene Chlorophosphonate and 2,2'-diphenylene Chlorothiophosphonate. Experienia, 32, 1111. http://dx.doi.org/10.1007/BF01927572

[5] Manne, P.N., Deshmukh, S.D., Rao, N.G.N., Dodale, H.G., Tikar, S.N. and Nimbalkar, S.A. (2000) Efficacy of Some Insecticides against Helicoverpa armigera (HUB). Pestology, 34, 65.

[6] Hendlin, D., Stapley, E.O., Jackson, M., Wallick, H., Miller, A.K., Woll, F.J., Miller, T.W., Chaiet, L., Kahan, F.M., Foltz, E.L., Woodruff, H.B., Mata, J.M., Hernandez, S. and Mochales, S. (1969) Phosphonomycin a New Antibiotic Produced by Strains of Streptomyces. Science, 166, 122-123. http://dx.doi.org/10.1126/science.166.3901.122

[7] Polozov, A.M. and Cremer, S.E. (2002) Synthesis of 2H-1,2-Oxaphosphorin 2-oxides. Journal of Organometallic Chemistry, 646, 153-160. http://dx.doi.org/10.1016/S0022-328X(01)01207-4

[8] Wu, X., Lei, X. and Fu, Z.F. (2003) Rabies Virus Nucleoprotein Is Phosphorylated by Cellular Casein Kinase II. Biochemical and Biophysical Research Communication, 304, 333-338.

[9] Gholivand, K., Shariatinia, Z., Mahzouni, H.R. and Amiri, S. (2007) Phosphorus Heterocycles: Synthesis, Spectroscopic Study and X-Ray Crystallography of Some New Diazaphosphorinanes. Structural Chemistry, 18, 653-660. http://dx.doi.org/10.1007/s11224-007-9197-3

[10] Page, D.S. (1981) Principles of Biological Chemistry. 2nd Edition, Willard Grant Press, Boston.

[11] Hugcl, H.M. and Jackson, N. (2012) Special Feature Organo-Fluorine Chemical Science. Applied Sciences, 2, 558-565. http://dx.doi.org/10.3390/app2020558

[12] Ismail, F.M.D. (2002) Important Fluorinated Drugs in Experimental and Clinical Use. Journal of Fluorine Chemistry, 118, 27-33. http://dx.doi.org/10.1016/S0022-1139(02)00201-4

[13] Elliott, A.J. (1995) Chemistry of Organic Fluorine Compounds. In: Hudlicky, M. and Pavlath, A.E., Eds., Chemistry of Organic Fluorine Compounds II: A Critical Review, American Chemical Society, Washington DC, 1119-1125.

[14] Dolbier Jr., W.R. (2005) Fluorine Chemistry at the Millennium. Journal of Fluorine Chemistry, 126, 157-163. http://dx.doi.org/10.1016/j.jfluchem.2004.09.033

[15] Smart, B.E. (2001) Fluorine Substituent Effects (on Bioactivity). Journal of Fluorine Chemistry, 109, 3-11. http://dx.doi.org/10.1016/S0022-1139(01)00375-X

[16] Ojima, I., McCarthy, J.R. and Welch, J.T., Eds. (1996) Biomedical Frontiers of Fluorine Chemistry. American Chemical Society, Washington DC. http://dx.doi.org/10.1021/bk-1996-0639

[17] Al-Romaizan, A.N., Abdel-Rahman, R.M. and Makki, M.S.T. (2014) Synthesis of New Fluorine/Phosphorus Substituted 6-(2'-Amino phenyl)-3-thioxo-1,2,4-triazin-5 (2H, 4H) One and Their Related Alkylated Systems as Molluscicidal Agent as against the Snails Responsible for Bilharziasis Diseases. International Journal of Organic Chemistry, 4, 154-168.

[18] Abdel-Rahman, R.M. (2001) Role of Uncondenced1,2,4-triazinecompounds and Related Heterobicyclic Systems as Therapeutic Agents. pharmazie, 56, 18-30.

[19] Abdel-Rahman, R.M. (2001) Role of Uncondensed 1,2,4-Triazinederivatives as Biological Plant Protection Agents. 
Pharmazie, 56, 195-212.

[20] Abdel-Rahman, R.M. (1999) Synthesis and Chemistry of Fluorine Containing 1,2,4-triazines. Pharmazie, 54, 791-804.

[21] Abdel-Rahman, R.M. (2002) Synthesis of New Phosphaheterobicyclic Systems Containing 1,2,4-Triazine Moiety Part IX; Straightforward Synthesis of New Fluorine Bearing 5-Phospha-1,2,4-triazin/1,2,4-triazepine-3-thions-part X. Trends in Heterocyclic Chemistry, 8, 187-195.

[22] Treov, K.D. (2006) Chemistry and Application of H-Phosphonates. El-Services, Amsterdam, 256.

[23] Cherkasov, R.A. and Galki, V.I. (1998) The Kabachnik-Fields Reaction: Synthetic Potential and the Problem of the Mechanism. Russian Chemical Reviews, 67, 857-940. http://dx.doi.org/10.1070/RC1998v067n10ABEH000421

[24] Annie Bligh, S.W., Mc Grath, C.M., Failla, S. and Finocchiaro, P. (1996) $\alpha$-Aminophosphate Monoester in One Step. Phosphorus, Sulfured Silicon, 118, 189-194.

[25] El-Sayed Ali, T. (2008) Synthesis and Characterization of Novel Bis-( $\alpha$-Aminophosphonates) with Terminal Chromone Moieties. ARKIVOC, 2, 71-79.

[26] El-Sayed Ali, T., Abdul-Ghaffar, S.A., El-Mahdy, K.M. and Abdel-Karim, S.M. (2013) Synthesis, Characterization, and Antimicrobial Activity of Some New Phosphorus Macrocyclic Compounds Containing Pyrazole Rings. Turkish Journal of Chemistry, 37, 160-169.

[27] Abdel-Rahman, R.M. and Ali, T.E. (2013) Synthesis and Biological Evaluation of Some New Polyfluorinated-4Thiazolidinone and $\alpha$-Amino Phosphonic Acid Derivatives. Monatshefte fur Chemie, 144, 1243-1252.

[28] Chakravarty, S. and Mishra, R.K. (1994) Spectrophotometric Determination of Phosphorus with N,N’-Diphenylbenzamidine and Methylen-Blue. Journal of Indian Chemical Society, 71, 717-719.

[29] Bukowski, L. (2001) Some Reactions of 2-cyanomethyl-3-methyl-3H-imidazo[4,5-b]pyridine with Isothiocyanates. Antituberculotic Activity of the Obtained Compounds. Pharmazie, 56, 23-27.

[30] Abdel-Rahman, R.M. and Abdel-Monem, W.R. (2007) Chemical Reactivity of 3-Hydrazino-5,6-diphenyl-1,2,4-triazine towards $\pi$-Acceptors Activated Carbonitriles. Indian Journal of Chemistry, 46B, 838-846.

[31] Abdel-Rahman, R.M. (1988) Reactions of 3-Hydrazino-5,6-diphenyl-1,2,4-triazine with Unsymmetrical 1,3-bicarbonyl Compounds: Synthesis of Some New 3-(3',5'-Disubstituted pyrazol-1'-yl(-5-6-diphenyl-1,2,4-triazines of Their Antimicrobial Activity. Indian Journal of Chemistry, 27B, 548-553.

[32] Zaher, H.A., Abdel-Rahman, R.M. and Abdel-Halim, A.M. (1987) Reactions of 3-Hydrazino-5,6-diphenyl-1,2,4-triazine with $\alpha, \beta$-Bifunctional Compounds. Indian Journal of Chemistry, 26B, 110-115.

[33] Gamal, A.A. (1997) Studies on 3-Amino-1,2,4-triazole. Journal of the Indian Chemical Society, 74, 624-625.

[34] El-Gendy, Z., Morsy, J.M., Allimony, H.A., Abdel-Monem, W.R. and Abdel-Rahman, R.M. (2001) Synthesis of Heterobicyclic Nitrogen Systems Bearing the 1,2,4-triazine Moiety as Anti-HIV and Anticancer Drugs, Part III. Pharmazie, 56, 376-382.

[35] El-Gendy, Z., Morsy, J.M., Allimony, H.A., Abdel-Monem, W.R. and Abdel-Rahman, R.M. (2003) Synthesis of Heterobicyclic Nitrogen Systems Bearing a 1,2,4-Triazine Moiety as Anticancer Drugs, Part IV. Phosphorus, Sulfur and Silicon, 179, 2055-2071. http://dx.doi.org/10.1080/10426500390228738

[36] Haddadin, M.J. and Hassner, A. (1973) Cycloaddition Reactions. XIV. Thermal and Photochemical Reactions of Some Bicyclic Aziridine Enol Ethers. Journal of Organic Chemistry, 38, 3466-3471. http://dx.doi.org/10.1021/jo00960a005

[37] Abdel-Rahman, R.M. and El-Gendy, Z. (1989) Synthesis of Some New1,2,4-Benzotriazine Derivatives from 2-Methyl Benzoxazole. Indian Journal of Chemistry, 28B, 1072-1076.

[38] Buchannan, G.W., Whitman, R.H. and Malaiyandi, M. (1982) A Carbon-13 Nuclear Magnetic Resonance Spectral Investigation of Substituted Triphenyl Phosphates. Organic Magnetic Resonance, 19, 98-101. http://dx.doi.org/10.1002/mrc.1270190211

[39] Al-Ravi, J.M.A., Behnam, G.O., Naceur, A. and Kruemer, R. (1985) Carbon-13 Chemical Shift Assignment of Some Organophosphorus Compounds. IV-2-oxo- and 2-thio-2-phenoxy-1,3,2-Diazaphosphorinanes and Related P(IV) Compounds. Magnetic Resonance in Chemistry, 23,728-731. http://dx.doi.org/10.1002/mrc.1260230910

[40] Gorenstein, D.G. and Kar, D.J. (1977) Effect of Bond Angle Distortion on Torsional Potentials. Ab Initio and CNDO/2 Calculations on Dimethoxymethane and Dimethyl Phosphate. Journal of the American Chemical Society, 99, 672-677.

[41] Reddy, C.D., Reddy, G.T. and Reddy, M.S. (1993) ${ }^{1} \mathrm{H},{ }^{13} \mathrm{C}$ and ${ }^{31} \mathrm{P}$ NMR Studies of 2-(Substituted Phenoxy) 2, 3-Dihydro-1H-Naphtho-[1, 8-de]-1, 3,2-Diazaphosphorine-2-Oxides. Asian Journal of Chemistry, 5, 291-295.

[42] Clercq, E.D. (2002) New Developments in Anti-HIV Chemotherapy. Biochemical et Biophysica Acta, 1587, $258-275$.

[43] Xie, L., Takeuchi, Y., Mark, L. and Lee, K.H. (1999) Anti-AIDS Agents. 37. Synthesis and Structure-Activity Relationships of (3’R,4'R)-(+)-cis-Khellactone Derivatives as Novel Potent Anti-HIV Agents. Journal of Medicinal Chemistry, 42, 2662-20877. http://dx.doi.org/10.1021/jm9900624 
[44] Connoly, K.J. and Hammer, S.M. (1992) Comparative Pharmacokinetics, Distributions in Tissue, and Interactions with Blood Proteins of Conventional and Sterically Stabilized Liposomes Containing 2',3'-Dideoxyinosine. Antimicrobial Agents and Chemotherapy, 36, 245-254.

[45] Mitsuya, H., Weinhold, K.J., Fuman, P.A., Clair, M.H., Lehrman, S.N., Gallo, R.C., Bolognesi, D., Barry, D.W. and Broder, S. (1985) 3'-Azido-3'-deoxythymidine (BW A509U): An Antiviral Agent That Inhibits the Infectivity and Cytopathic Effect of Human T-lymphotropic Virus Type III/Lymphadenopathy-Associated Virus in Vitro. Proceedings of the National Academy of Sciences of the United States of America, 82, 709670-707100.

[46] Mitsuya, H. and Broder, S. (1986) Inhibition of the in Vitro Infectivity Andcytopathic Effect of Human T-Lymphotrophic Virus Type III/Lymphadenopathy-Associated Virus (HTLV-III/LAV) by 2',3'-dideoxynucleosides. Proceedings of the National Academy of Sciences of the United States of America, 83, 1911-1915. http://dx.doi.org/10.1073/pnas.83.6.1911

[47] Bozzette, S.A. and Richman, D.D. (1990) Salvage Therapy for Zidovudine-Intolerant HIV-Infected Patients with Alternating and Intermittent Regimens of Zidovudine and Dideoxycytidine. The American Journal of Medicine, 88, S24S26. http://dx.doi.org/10.1016/0002-9343(90)90418-D

[48] Dunkel, L., Cross, A., Martin, R., Brown, M. and Murray, H., (1990) Dose-Escalating Study of Safety and Efficacy of Dideoxydidehydrothymidine (d4T) for HIV Infection. Antiviral Research, 13, 116. http://dx.doi.org/10.1016/0166-3542(90)90217-U

[49] Romero, D.L., Busso, M., Tan, C.K., Reusser, F., Palmer, J.R., Poppe, S.M., Aristoff, P.A., Downey, K.M., So, A.G., Resnick, L. and Tarpley, W.G. (1991) Non-Nucleoside Reverse Transcriptase Inhibitors That Potently and Specifically Block Human Immunodeficiency Virus Type 1 Replication. Proceedings of the National Academy of Sciences of the United States of America, 88, 8806-8810. http://dx.doi.org/10.1073/pnas.88.19.8806

[50] Srivastava, A.K., Khan, A.A. and Shakil, M. (2001) Quantitative Structure Activity Relationship (QSAR) Studies on Anti-HIV-1 and Cytotoxic Arylpyrrolylsulfones. Journal of the Indian Chemical Society, 78, 154-157.

[51] Lu, C. and Li, A.P. (2010) Enzyme Inhibition in Drug Discovery and Development: The Good and the Bad. Wiley, OU143, E 605.

[52] Sergei, V., Gulni, K., Elena, A. and Michael, E. (2010) Enzyme Inhibition in Drug Discovery and Developmental Edited, Lu \& Li. Wiley \& Sons, Inc., New York, 749.

[53] Weislow, O.W., Kiser, R., Fine, D., Bader, J., Shoemaker, R.N. and Boyd, M.R. (1989) New Soluble Formazan Assay for HIV-1 Cytopathiceffects, Application to High Flux Screening of Synthetic and Natural Products for AIDS-Antiviral Activity. Journal of the National Cancer Institute, 81, 577-586. http://dx.doi.org/10.1093/jnci/81.8.577

[54] Chaouni, B.A., Galtier, C., Allouchi, H., Kherbeche, A., Chavignon, O., Teulade, J.C., Witvrouw, M., Pannecouque, C., Snoeck, R., Andrei, G., Balzarini, J., De Clercq, E., Fauvelle, F., Enguehard, C. and Gueiffier, A. (2001) 3-Benzamido, Ureido and Thioureidimidazo[1,2-a]Pyridine Derivatives as Potential Antiviral Agents. Chemical and Pharmaceutical Bulletin, 49, 1631-1635. http://dx.doi.org/10.1248/cpb.49.1631 
Scientific Research Publishing (SCIRP) is one of the largest Open Access journal publishers. It is currently publishing more than 200 open access, online, peer-reviewed journals covering a wide range of academic disciplines. SCIRP serves the worldwide academic communities and contributes to the progress and application of science with its publication.

Other selected journals from SCIRP are listed as below. Submit your manuscript to us via either submit@scirp.org or Online Submission Portal.
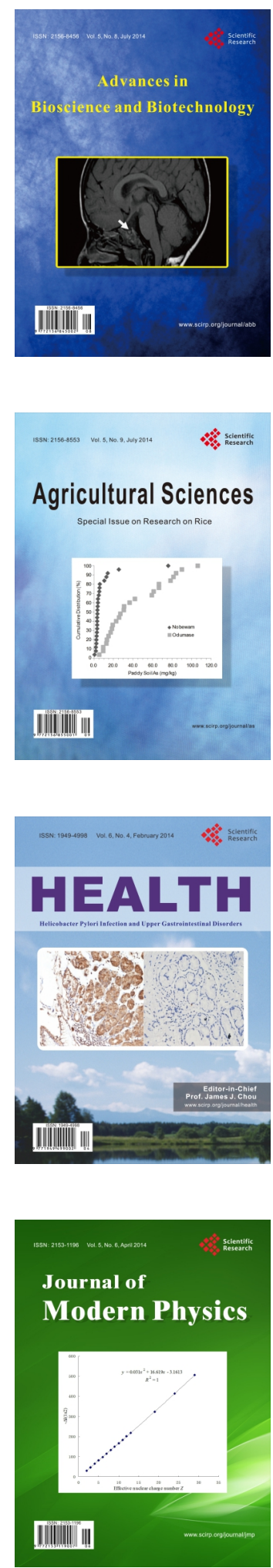
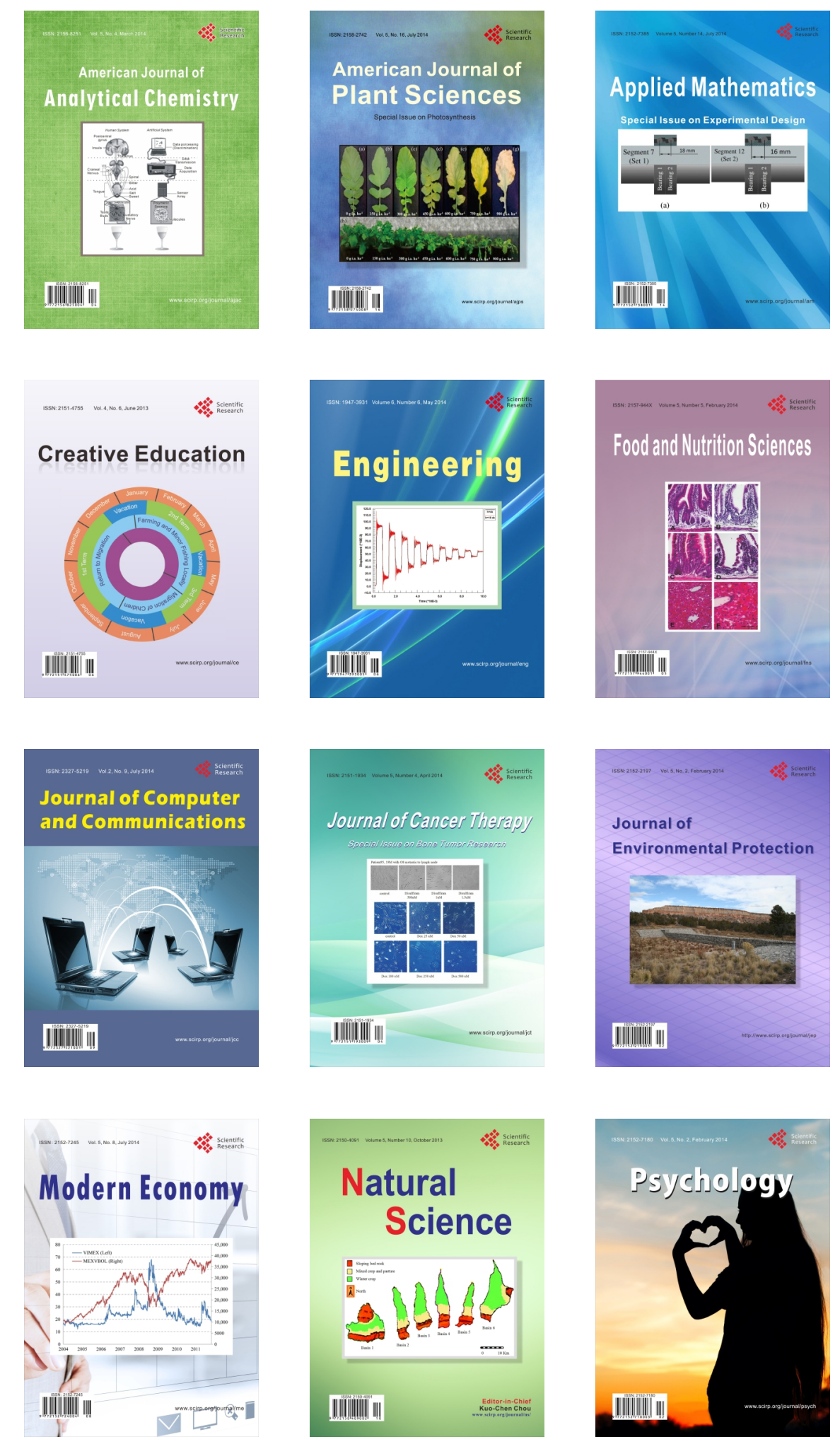